\title{
Cosmological implications of electromagnetically interacting dark matter: milli-charged particles and atoms with singly and doubly charged dark matter
}

\author{
Gautham A P ${ }^{a}$ Shiv Sethi ${ }^{b}$ \\ ${ }^{a}$ Indian Institute of Technology Madras(IITM),Chennai, India \\ ${ }^{b}$ Raman Research Institute(RRI),Bangalore, India \\ E-mail: apgautham@gmail.com, sethi@rri.res.in
}

\begin{abstract}
While the behavior of the dominant component of the dark matter is reasonably well established by cosmological observables, its particle nature and interactions with the rest of the matter are not known. We consider three dark matter models that admit electromagnetic interaction between baryons and dark matter: (a) milli-charged particle (CCDM) of charge $q_{\mathrm{ccdm}}$ and mass $m_{\mathrm{ccdm}}$, (b) a neutral atom of two charged particles of mass $m_{\mathrm{dd}}$ (DD), and (c) a neutral atom of doubly charged particle and helium nucleus (HeD). We derive and discuss in detail the formation, stability, and interaction of these atoms with baryons. We derive the implications of this new interaction in the tight-coupling approximation, which allows us to analytically gauge their impact on the matter power spectrum and CMB anisotropy. We incorporate this new interaction into the publicly-available code CLASS to obtain numerical results. We compare our results with Planck 2018 data to constrain the fraction of interacting dark matter. For the range of allowed astrophysical parameters, the HeD atom yields the results of $\Lambda \mathrm{CDM}$ model for $k<1 \mathrm{Mpc}^{-1}$, and hence its fraction is not constrained by CMB anisotropy data which is sensitive to $k<0.2 \mathrm{Mpc}^{-1}$. For $m_{\mathrm{dd}} \gtrsim 25 \mathrm{GeV}$, the DD atom is also not constrained by CMB data. For $m_{\mathrm{dd}}=10 \mathrm{GeV}$, the CMB data constrains the fraction of DD atoms to be smaller than $4 \%$ of the total CDM component. For $q_{\mathrm{ccdm}}=10^{-6} e$ and $m_{\mathrm{ccdm}}=50 \mathrm{MeV}$, the CCDM fraction is constrained to be less than $1 \%$.
\end{abstract}




\section{Contents}

1 Introduction $\quad 2$

2 Dark matter models with electromagnetic interaction 3

2.1 Milli-Charged Dark Matter 3

2.2 Neutral atoms with charged dark matter 4

2.2.1 Formation of $\mathrm{HeD}$ and DD atoms 4

2.2.2 Charged particle scattering off neutral atoms: different approximations 6

2.3 The rate of momentum loss 8

3 Evolution of coupled dark matter-baryon density and velocity perturbations

3.1 Dynamics of baryon-photon-dark matter fluid: tight-coupling approximation 10

$\begin{array}{lll}3.2 & \text { Matter power spectrum and CMB anisotropies } & 11\end{array}$

$\begin{array}{lll}\text { 3.2.1 Numerical implimentation in CLASS } & 13\end{array}$

4 Matter power spectrum $\quad 14$

$\begin{array}{lll}4.1 & \text { CMB anisotropies } & 16\end{array}$

5 Comparison with data 17

6 Conclusion and future prospects $\quad 19$ 


\section{Introduction}

The nature of dark matter remains a mystery even though the existence of this component has been well established by many observations covering a wide range of mass scales - from dwarf galaxies to clusters of galaxies - and different epochs of the universe through cosmological probes. This list includes cosmic microwave background (CMB) anisotropy experiments $[2$, $40,56,60]$, large scale structure surveys $[16,62,63]$, the study of the galaxy rotation curves [14], cosmological weak gravitational lensing observations [12, 24], etc.

The Weakly Interacting Massive Particle (WIMP) is normally considered the leading candidate for the cold dark matter (CDM) in the universe. Its popularity is partly inspired by the well-known WIMP miracle[25]. The supersymmetric extension of the standard model of particle physics allows for a stable particle in the mass range $10-1000 \mathrm{GeV}$ with selfannihilation cross-section $\langle\sigma v\rangle \simeq 10^{-26} \mathrm{~cm}^{3} \mathrm{~s}^{-1}$; these parameters predict the abundance of cold dark matter in the observed range. This theoretical insight has inspired many direct, indirect and collider searches of the WIMP [1, 4-6, 8, 10, 13, 37, 39]. Even though these searches have not yet succeeded, the sensitivity of the direct detection experiments, LUX and XENON, has improved considerably in the recent years [7, 9].

The cold dark matter paradigm based on WIMP has long-standing astrophysical issues: core-cusp problem [27, 55, 66], missing satellites of the Milky way [45, 51], "too big to fail" issue $[20,38]$. All these issues probably indicate a need to go beyond the WIMP model and to consider alternative candidates, which differ from the model on galactic scales but must reproduce its success on cosmological scales.

The focus of this paper is to study cosmological implications of dark matter particles with electromagnetic interactions. Such models were proposed before precision cosmological data became available $[28,29]$. In such models, charged particles that survive annihilation in the early universe could form atoms with protons and helium nuclei and provide the observed mass density of cold dark matter in the universe. Many such models were ruled out and revised as better astrophysical and cosmological data became available (e.g. [18, 22, 23, 26, 34, 46, 67]). The current cosmological data clearly rules out a dark matter particle with electronic charge unless it is very heavy. One possible extension of this idea was a neutral atom formed with the proton and a negatively charged dark matter particle (e.g. [28]). Such an atom is not stable to charge-exchange reaction and therefore does not remain neutral as the universe evolves (for details of charge exchange reactions see e.g. [32]).

We consider three models for our study. The first model posits the presence of a millicharged dark matter particle (CCDM model, e.g. [23, 33, 47]). The model-parameterized by the magnitude of milli-charge and the mass of the dark matter particle - has been extensively studied against cosmological data (e.g. [47]). The second model assumes the presence of two dark matter particles of positive and negative electronic charge which recombine to form a neutral atom (DD model). The stability of this atom to charge exchange reaction constrain the masses of charged particles. The third model, which is a natural extension of the protondark matter atom, is based on a doubly charged dark matter particle (HeD model, see [44] for theoretical motivation for such a scenario) which recombines with a helium nucleus to form a neutral atom. The mass of this particle must be much larger than the helium nucleus to ensure, (a) only a small fraction of helium nuclei recombine with the dark matter particle to satisfy nucleosynthesis bounds, and (b) the atom is stable to charge-exchange reactions. We discuss in the detail the recombination, ionization, and charge-exchange stability of such atoms, in addition to computing the scattering of such atoms off baryons. 
One of the motivation of studying such models is that these models might allow, for some parameter range, smaller matter power at small scales as compared to the WIMP model. So some of these models can be viewed as complementary to other existing models to explain the missing small scale power (e.g. warm dark matter (WDM) model, [31, 65] and other models such as LFDM, CHDM, or ULA; for details see e.g. [41, 59, 61]) The milli-charged dark matter model has recently been invoked to explain the EDGES results [11, 19, 35, 47].

In the next section, we discuss the three dark matter models we consider and study their formation, stability, and interaction with baryons. In section 3, we consider the evolution of coupled baryon-dark matter system in linear perturbation theory relevant for the early universe. In particular, we show how the new interaction can be incorporated within the framework of tight-coupling approximation, which allows us to gauge the impact of the new interaction on cosmological observables. We also discuss how this approximation allows us to numerically implement the altered evolution of coupled fluids. In section 4 , we study the impact of the new interaction on cosmological observables such as the matter power spectrum and $\mathrm{CMB}$ anisotropies and also provide results of MCMC analysis which assesses the viability of the new models against the Planck data. In section 6 we summarize and discuss our results.

For computing the matter power spectra for the proposed models, we assume a spatially flat universe with its best-fit cosmological parameters estimated by Planck [56]: $\Omega_{b}=0.049$, $\Omega_{\mathrm{dm}}=0.254$ (this corresponds to the total cold dark matter content of the universe in our case as we consider models where there are two components of the dark matter), and $h=0.67$.

\section{Dark matter models with electromagnetic interaction}

In this section we discuss in detail the formation of atoms with charged dark matter and their interaction with baryons.

\subsection{Milli-Charged Dark Matter}

The milli-Charged dark matter can interact with charged baryons via coulomb interaction. Assuming baryons and dark matter particles to be at different temperatures and averaging over the thermal distribution, we get the interaction rate to be (e.g. [47]):

$$
\frac{1}{\tau_{\mathrm{bd}}}=n_{\mathrm{ccdm}} \frac{4 \pi}{3} \sqrt{\frac{2}{\pi}}\left(\frac{e q_{\mathrm{ccdm}}}{\mu}\right)^{2} \frac{m_{\mathrm{ccdm}}}{m_{\mathrm{ccdm}}+m} \log _{e} \Lambda\left[3 k_{b}\left(\frac{T}{m}+\frac{T_{\mathrm{ccdm}}}{m_{\mathrm{ccdm}}}\right)\right]^{-\frac{3}{2}}
$$

Here $m$ and $T$ stand for the mass and temperature of either electron, proton, or helium nucleus while $m_{\mathrm{ccdm}}, T_{\mathrm{ccdm}}$, and $q_{\mathrm{ccdm}}$ denote the mass, temperature, and charge, respectively, of the milli-charged dark matter particle. $\mu$ is the reduced mass of $m$ and $m_{c c d m}$ and $\Lambda$ is the usual coulomb logarithm. If $f_{\mathrm{ccdm}}$ is the fraction of cold dark matter in the form of milli-charged particles, the mass density and the number density of CCDM dark matter are: $\rho_{\mathrm{ccdm}}=f_{\mathrm{ccdm}} \Omega_{\mathrm{dm}} \rho_{c}$ and $n_{\mathrm{ccdm}}=f_{\mathrm{ccdm}} \Omega_{\mathrm{dm}} \rho_{c} / m_{\mathrm{ccdm}}$. In the expression above we have assumed the dark matter to be the target. For the reverse reaction, the time scale: $\tau_{\mathrm{db}}=\tau_{\mathrm{bd}} \rho_{\mathrm{ccdm}} / \rho_{b}$, as discussed in section 2.3.

In deriving Eq. (2.1), we have neglected the bulk peculiar velocity and, as already noted above, the dark Matter particles obey Maxwell-Boltzmann distribution. Eq. (2.1) shows that the electron-DM interaction is the dominant scattering process. As the time scale of equilibriation between electron, proton, and Helium nuclei is much shorter than the expansion rate, they share a common temperature. For the purposes of computing CMB anisotropies, 
this means that when electron-DM interaction time scale is shorter than the expansion time, the dark matter particles can be assumed to be coupled to the entire baryonic fluid.

Eq. (2.1) shows that the interaction rate (the inverse of interaction time) falls as $a^{-3 / 2}$ while the expansion rate, $H$, drops as $a^{-2}$ in the radiation-dominated era. Therefore, the coulomb interaction becomes more important at later times; the ratio of the interaction and expansion rate is given in Eq. (2.16). At high redshifts, the DM and baryons are decoupled, which means that their initial temperatures could be different. However, in this work, we assume these temperatures to be the same.

\subsection{Neutral atoms with charged dark matter}

It is well known that a free dark matter particle of electronic charge is incompatible with $\mathrm{CMB}$ and large scale clustering data because its strong interaction with baryons results in a coupled baryon-photon-dark matter fluid ${ }^{1}$. One possible way to reduce interaction between dark matter and baryons is to consider cases in which dark matter particles form atoms with either another dark matter particle or with baryonic particles (protons or Helium nuclei ${ }^{2}$ ).

An important consideration in such cases is that the neutral atom be stable to chargeexchange reactions (to be discussed in more detail below). For instance, the dark matter carrying electronic charge can form a neutral atom with protons. However, such an atom is unstable to charge-exchange reaction in which the proton is replaced by the helium nucleus in the atom, yielding an atom with a net charge.

This motivates us to consider two cases: (a) a neutral atom formed with a doubly-charged DM particle of mass $m_{\text {hed }}$ with Helium nucleus such that $m_{\text {hed }}>m_{\text {he }}{ }^{3}$ and (b) an atom formed with two singly charged DM particles of masses $m_{1}$ and $m_{2}$ such that $m_{1}, m_{2} \gg m_{\text {he }}$ or both the particles are much heavier than the helium nucleus. The two masses $m_{1}, m_{2}$ are otherwise unconstrained and we consider the case when $m_{1}=m_{2}$; we denote this common mass as $m_{\mathrm{dd}}$.

\subsubsection{Formation of $\mathrm{HeD}$ and $\mathrm{DD}$ atoms}

The three most important physical processes for the formation and destruction of these atoms are: (a) photoionization, (b) recombination, and (c) charge exchange.

The cross section of charge-exchange reaction is on the same order of magnitude as the elastic scattering cross section between the charged particle and the neutral atom, discussed in the next subsection, if the reaction is exothermic or the resultant atom is more stable (e.g. [32]).

Protons and Helium nuclei can form atoms with negatively-charged dark matter particles. If dark matter is singly charged then protons can form neutral atoms with dark matter but charge-exchange reaction replaces proton with helium nucleus. This reaction is energetically favored as it results in a more stable atom. Using the analysis of the next section it can be shown that the rate of this reaction exceeds the expansion rate for $z>10^{5}$ therefore it is not possible to sustain a neutral dark matter atom with a single proton. This is our primary motivation for considering a doubly charged dark matter particle.

\footnotetext{
${ }^{1}$ If the dark matter particle is very heavy, this constraint can be obviated because the dark matter could remain uncoupled from baryons up to $z \simeq 1000$; the lower limit on the mass of such a dark matter particle can be computed from Eq. (2.1) (see e.g. [43])

${ }^{2}$ Primordial nucleosynthesis also produces a small fraction, $\simeq 10^{-5}$ of baryons, of ${ }^{3} \mathrm{He}$. In this paper, the helium nucleus always refers to ${ }^{4} \mathrm{He}$, whose mass abundance is nearly $25 \%$ of baryons.

${ }^{3}$ As discussed in section 4 , we require $m_{\text {hed }} \gg m_{\text {he }}$ to satisfy nucleosynthesis and CMB constraints.
} 
Two dark matter particles carrying electronic charge can form a stable neutral atom only if the two equal mass particles are much heavier than the helium nucleus (to be discussed later). If these particles are lighter than the Helium nucleus, charge-exchange reaction would replace one of the particles with helium nucleus.

If the dark matter is doubly charged, it could form two different neutral atoms: ppD or $\mathrm{HeD}$. The charge-exchange reaction would turn the first atom into a more stable, $\mathrm{pHeD}$, and therefore it would not remain neutral. The only stable neutral atom is HeD. Therefore, if the dark matter atom is to remain neutral during the pre-recombination phase, it is imperative that a large fraction of the dark matter particles are captured to form HeD before ppD could form during the evolution of the universe.

We compute the rate of formation of hydrogenic atoms $\mathrm{pD}$ and $\mathrm{HeD}$ to address this issue. All quantities are scaled with respect to corresponding cross sections for the hydrogen atom.

Binding energy: For $m_{\mathrm{dm}} \gg m_{\mathrm{He}}$, the binding energies of the $\mathrm{pD}$ and $\mathrm{HeD}$ atoms are $Z^{2}\left(m_{p} / m_{e}\right)$ Ry and $Z^{4}\left(m_{\mathrm{He}^{4}} / m_{e}\right) \mathrm{Ry}$, respectively. For the DD atom, the binding energy is $\left(m_{\mathrm{dd}} / 2 m_{e}\right)$ Ry. Here $1 \mathrm{Ry}=13.6 \mathrm{eV}$ is the binding energy of the hydrogen atom.

Ionization cross section: The ionization cross section of different atoms can be computed from appropriate scaling of the cross section for hydrogen atom. We compute these cross sections at the ionization threshold of each species, where it is the maximum, and express them in terms of hydrogen ionization cross section. The ionization cross sections $\operatorname{are}^{4}$ :

$$
\begin{aligned}
\sigma_{\mathrm{pD}}^{\mathrm{ion}}\left(\nu_{\mathrm{pD}}\right) & =\frac{m_{e}^{2} \sigma_{\mathrm{H}}^{\mathrm{ion}}}{Z^{5} m_{p}^{2}} \\
\sigma_{\mathrm{HeD}}^{\mathrm{ion}}\left(\nu_{\mathrm{HeD}}\right) & =\frac{m_{e}^{2} \sigma_{\mathrm{H}}^{\text {ion }}}{Z^{8} m_{\mathrm{He}}^{2}} \\
\sigma_{\mathrm{DD}}^{\mathrm{ion}}\left(\nu_{\mathrm{DD}}\right) & =\frac{4 m_{e}^{2} \sigma_{\mathrm{H}}^{\text {ion }}}{m_{\mathrm{dd}}^{2}}
\end{aligned}
$$

Here $Z=2$ and $\sigma_{\mathrm{H}}^{\text {ion }}=6.3 \times 10^{-18} \mathrm{~cm}^{2}$ is the cross section of hydrogen at ionization threshold $\nu=13.6 \mathrm{eV}$.

Recombination cross section: Using Milne relation (e.g see [57] for details), the recombination cross sections can be computed from ionization cross sections. This gives:

$$
\begin{aligned}
\sigma_{\mathrm{pD}}^{\mathrm{rec}}(v) & \simeq \frac{\chi_{\mathrm{H}}^{2} \sigma_{\mathrm{H}}^{\text {ion }}}{Z m_{p}^{2} c^{2} v^{2}} \\
\sigma_{\mathrm{HeD}}^{\mathrm{rec}}(v) & \simeq \frac{\chi_{\mathrm{H}}^{2} \sigma_{\mathrm{H}}^{\text {ion }}}{m_{\mathrm{He}}^{2} c^{2} v^{2}} \\
\sigma_{\mathrm{DD}}^{\mathrm{rec}}(v) & \simeq \frac{2 \chi_{\mathrm{H}}^{2} \sigma_{\mathrm{H}}^{\text {ion }}}{m_{\mathrm{dd}}^{2} c^{2} v^{2}}
\end{aligned}
$$

Here $\chi_{\mathrm{H}}=13.6 \mathrm{eV}$ is the binding energy of hydrogen atom and $v$ is the relative velocity of the two recombining particles. We have assumed that this velocity is much smaller than the binding energy of respective atoms. A quantity of interest is the recombination coefficient, $\alpha(T)=\langle v \sigma(v)\rangle$, where the average is over thermal distribution of particles; all the species

\footnotetext{
${ }^{4}$ These cross-sections can be computed from positive-energy spherically-symmetric solutions of a hydrogenic atom, which are valid for the ionization process close to the threshold; for details see e.g. chapter IVb of [15] or section 148, problem 4 of [48].
} 
are in thermal equilibrium owing to the initial condition at temperature $T$ and therefore we could replace $m v^{2} \simeq 2 k T$ for each species.

The evolution of the ionization state of each species is given by:

$$
\frac{d n_{i}}{d t}=n_{\mathrm{dm}} n_{j} \alpha_{i}(T)-c n_{i} \int_{\nu_{i}}^{\infty} n_{\gamma}(\nu) \sigma_{i}(\nu) d \nu
$$

Here $n_{i}$ refers to the number density of either $\mathrm{pD}, \mathrm{HeD}$ or $\mathrm{DD}$ atoms. $n_{j}$ corresponds to the number density of either protons or helium nuclei or, for the case of DD atom, the number density of dark matter particles. $n_{\mathrm{dm}}$ is the number density of dark matter particles, which for the case of $D D$ atom is the same as $n_{j} . \nu_{i}, \sigma_{i}$, and $\alpha_{i}$ give ionization thresholds, ionization cross sections, and recombination coefficients for different atoms. At redshifts of interest, the main source of photoionization of these atoms is background blackbody radiation which gives the number density of ionizing photons per unit frequency, $n_{\gamma}=B_{\nu}(T) /(c h \nu)$, where $B_{\nu}(T)$ is the blackbody specific intensity at temperature $T$.

Eq. (2.8) can be solved numerically. We do not present numerical results here but outline the essential outcome of the solution of the equation. We first consider $\mathrm{pD}$ and $\mathrm{HeD}$ atoms. Our aim is two-fold: (a) to show that the recombination rates are large enough in comparison with expansion and ionization rates to allow the formation of these atoms, (b) the DM atoms preferably recombine with helium nuclei and not with protons. The rate of ionization at any redshift can be approximated as $B_{\nu_{i}}(T) \sigma_{i}\left(\nu_{i}\right) / h$, where $\nu_{i}$ corresponds to ionization threshold. We first compare the ionization rates of the two atoms at $z \simeq 2 \times 10^{8}$. At this redshift, the ionization threshold of $\mathrm{pD}$ atom corresponds to the peak of the Planckian. At the redshift, the ionization rate of $\mathrm{HeD}$ is smaller than $\mathrm{pD}$ by more than 10 orders of magnitude. While the former rate is smaller than both the expansion rate and recombination rate, the latter is much larger than both. Therefore, at this redshift, the rates are favourable to the formation of $\mathrm{HeD}$ atom while $\mathrm{pD}$ cannot form. We check that the conditions are such that nearly all the DM particles recombine to form $\mathrm{HeD}$ around this redshift. As $\mathrm{HeD}$ atom is more stable, this atom cannot be further altered by charge-exchange processes.

As noted above the DD atom is stable only if its binding energy is much larger than other possible atoms that can form $(\mathrm{pD}$ and $\mathrm{HeD})$. The ratio of binding energy of a singly charged DM particle forming an atom with helium nucleus to DD atom is $Z^{2} m_{\mathrm{He}} / m_{\mathrm{dd}}$. This requires the DM particle to be at least four times heavier than the helium nucleus. We verify that if the ratio of binding energies is 10 then nearly all the dark matter particles recombine to form $\mathrm{DD}$ atom before the formation of $\mathrm{HeD}$ atom.

We note that the recombination process cannot result in the capture of all the dark matter particles into neutral atoms, as the recombination process becomes inefficient after a majority of dark matter particles have already recombined (the first term of Eq. (2.8)). Therefore, this process will always leave a tiny fraction of free charged dark matter particles. The exact fraction of this residual depends on the parameters of the models and the details of the recombination process. If $\Delta \rho_{\mathrm{dm}} \ll \rho_{b}$, this will have negligible effect on cosmological observables such as CMB anisotropies. We neglect the impact of this residual charged component of dark matter in our study.

\subsubsection{Charged particle scattering off neutral atoms: different approximations}

We consider the scattering of charged particles with a neutral hydrogenic atom, of polarizability $\alpha_{0} \simeq a^{3}$, in its ground state, where $a$ is the Bohr radius of the ground state. For the two 
cases we consider, the Bohr radii are: $a_{\mathrm{hed}}=\hbar^{2} /\left(Z^{2} e^{2} m_{\mathrm{he}}\right)(Z=2)$ and $a_{\mathrm{dd}}=2 \hbar^{2} /\left(e^{2} m_{\mathrm{dd}}\right)$. (the Bohr radius for hydrogen atom: $a_{h}=\hbar^{2} /\left(e^{2} m_{\mathrm{e}}\right)$ )

We first consider this scattering in Born's approximation. This approximation is valid if the velocity of the charged particle is large as compared to the velocity of the lighter particle inside the atom, $k a \gg 1$. As all the species are in thermal equilibrium with each other, the kinetic energy of all the particles is $\simeq k_{\mathrm{B}} T$, this gives $k \simeq \sqrt{m k_{\mathrm{B}} T} / \hbar$ For the two cases we consider:

$$
\begin{aligned}
& k a_{\text {hed }} \simeq 2 \times 10^{-3}\left(\frac{T}{10^{6} \mathrm{~K}}\right)^{1 / 2} \\
& k a_{\mathrm{dd}} \simeq 4 \times 10^{-3}\left(\frac{m_{\mathrm{he}}}{m_{\mathrm{dd}}}\right)\left(\frac{T}{10^{6} \mathrm{~K}}\right)^{1 / 2}
\end{aligned}
$$

Therefore, except at very high temperatures, Born's approximation in not applicable to our analysis.

Another approximation commonly used in proton scattering off neutral atoms in interstellar medium is the semi-classical approximation which treats proton trajectory as classical. In this case, the cross section of scattering is $\simeq b_{0}^{2}$ where $b_{0} \simeq\left(e^{2} \alpha_{0} / m_{p} v^{2}\right)^{1 / 4}$. As $\alpha_{0} \simeq a^{3}$, $b_{0} \gg a$ at small temperatures $T<10^{4} \mathrm{~K}$. This approximation breaks down for electron scattering off neutral atoms. As this case requires $k b_{0} \gg 1$, we do not satisfy this condition except at very high temperature.

It is clear that many different approximations might be needed to study this scattering for a large range of temperatures. Generically, the neutral atom is coupled to charged particles at high redshifts and could decouple before recombination. One of our main aim is to determine the redshift of decoupling and there are interesting observational consequences when $z<10^{6}$. For the redshifts of interest, Eq. (2.10) shows that the suitable approximation is $k a \ll 1$. This is the limit of low energy scattering which can be studied using partial wave analysis. In this limit, only the $\ell=0$ partial wave contributes significantly ${ }^{5}$ and the cross section is independent of the energy and the scattering angle. For scattering of a proton/electron off a neutral atom in its ground state, the effective interaction potential $U=e^{2} \alpha / r^{4}$. The $s$-wave cross section of momentum exchange of these atoms with electrons, in the center-of-mass frame, is (see e.g. section 132 of [48]):

$$
\begin{aligned}
\sigma_{\mathrm{hed}} & \simeq 4 \pi a_{\mathrm{hed}}^{3} / a_{\mathrm{h}} \\
\sigma_{\mathrm{dd}} & \simeq 4 \pi a_{\mathrm{dd}}^{3} / a_{\mathrm{h}}
\end{aligned}
$$

Here $a_{\text {hed }}=\hbar^{2} /\left(Z^{2} e^{2} m_{\mathrm{he}}\right)$ and $a_{\mathrm{h}}=\hbar^{2} /\left(e^{2} m_{\mathrm{e}}\right)(Z=2)$ and $a_{\mathrm{dd}}=\hbar^{2} /\left(e^{2} m_{\mathrm{dd}}\right)$. For scattering of these atoms off protons, the mass of electron is replaced by the mass of proton in Eq. (2.12) and these cross sections are larger by a factor $\left(m_{p} / m_{e}\right)^{6}$. It should be noted that the cross sections are independent of the relative velocity. In the literature, atomic dark matter in the dark sector has been considered which might yield velocity-dependent cross section (e.g. $[26])$.

\footnotetext{
${ }^{5}$ the scattering amplitude for $\ell \neq 0$ scales as $(k a)^{2 \ell}$

${ }^{6}$ to be more exact, $m_{\text {he }}$ should be replaced by the reduced mass of the atom and $m_{e}$ or $m_{p}$ should be replaced by the reduced mass of the scattering particles. However, as the mass of the dark matter particle is much larger than the helium nucleus and the mass of the $\mathrm{HeD}$ atom is much larger than the proton mass, this is a good approximation
} 


\subsection{The rate of momentum loss}

For computing the time scales $\tau_{\mathrm{db}}$ and $\tau_{\mathrm{bd}}$ defined in section 3, we need to compute the rate at which the momentum is lost in the lab frame. This rate is defined as (e.g. [49]):

$$
\nu_{1}=\frac{v_{1}}{p} \frac{d p}{d l}
$$

Here $p$ is the momentum of the incident particle and $v_{1}$ its velocity in the lab frame. The target particle is assumed to be at rest in this frame so $v_{1}$ is the relative velocity between the two particles. $d p / d l$ the loss of momentum of the incident particle per unit length. It can be shown that:

$$
\nu_{1}=\frac{n_{2} v_{1} m_{2}}{\left(m_{1}+m_{2}\right)} \frac{d \sigma}{d \Omega}(\chi)(1-\cos \chi)
$$

Here $n_{2}$ and $m_{2}$ are the number density and the mass of the target particle, respectively. $\chi$ is the angle of scattering and $d \sigma / d \Omega$ is the differential cross section of scattering in the center of mass frame. Integrating Eq. (2.14) over the solid angle and averaging over the distribution of $v_{1}$ we obtain the final expression of the rate of momentum loss. Similarly, we can obtain $\nu_{2}$, the rate of the inverse process. It should be noted that $\nu_{1} / \nu_{2}=\rho_{2} / \rho_{1}$, where $\rho=n m$ is the mass density. Using Eqs. (3.4) and (3.2), it can readily be shown that the rate of momentum exchange between the two particles per unit volume $-\rho_{c} \dot{\theta_{c}}$ and $\rho_{b} \dot{\theta_{b}}$-is the same for the forward and the inverse process, as required by the conservation of momentum.

Eq. (2.14) allows us to compute the time scales $\tau_{\mathrm{bd}}$ and $\tau_{\mathrm{db}}$ which are inverse of the rate of momentum loss. Eq. (2.1) gives $\tau_{\text {bd }}$ for coulomb interaction.

For the other two models we discuss, the cross section is independent of velocity and the angle of scattering (Eq. (2.12)). For these cases, averaging over the thermal distribution of velocity, we get:

$$
\tau_{\mathrm{db}}^{-1}=\frac{2}{\pi^{1 / 2}}\left(\frac{n_{b} m}{m+m_{\mathrm{dm}}}\right) \sigma\left[\frac{2 k_{b} T}{m_{r}}\right]^{1 / 2}
$$

Here $n_{\mathrm{dm}}$ and $m_{\mathrm{dm}}$ correspond to number density and mass of either the DD or HeD atom. $m$ is the mass of either electron, proton, or Helium nucleus and $\sigma$ is the relevant cross section. $m_{r}$ is the reduced mass of the scattering particles. As discussed above, $\tau_{\mathrm{bd}}^{-1}=\tau_{\mathrm{db}}^{-1} \rho_{\mathrm{dm}} / \rho_{b}$.

We note that the scattering cross section might not correspond to a single process but could be an effective cross section as the dark matter particle (which refers to either CCDM particle or dark matter atom) interacts electromagnetically with free electrons, protons and helium nuclei. The cross section of interaction between the dark matter and these particles is not the same. The impact of all these interactions can be captured by defining an effective

time of interaction, $\tau_{\mathrm{db}}^{-1}=\sum_{i} 1 / \tau_{\mathrm{di}}$, where $i$ runs over these species, because all these particles share the momentum exchange with the dark matter particle. This also allows us to compute an effective scattering cross section $\sigma_{\mathrm{db}}$ in terms of the scattering cross sections with different charged particles. Similarly, $\sigma_{\mathrm{bd}}$ can also be defined. In our case, the scattering is dominated by a single process for each case: CCDM-electron, DD-proton, and HeD-proton.

The dark matter and baryons get strongly coupled when their interaction time scales become comparable to the expansion rate. The ratio of the interaction rate and the expansion 
rate, $H$, is:

$$
\begin{aligned}
& \frac{1}{\tau_{\mathrm{db}}^{\mathrm{ccdm}} H} \simeq 0.1\left(\frac{5 \mathrm{MeV}}{m_{\mathrm{ccdm}}}\right)\left(\frac{q_{\mathrm{ccdm}}}{10^{-6} e}\right)^{2}\left(\frac{5 \times 10^{4}}{z}\right)^{1 / 2} \\
& \frac{1}{\tau_{\mathrm{db}}^{\mathrm{dd} H}} \simeq 6 \times 10^{-2}\left(\frac{25 \mathrm{GeV}}{m_{\mathrm{dd}}}\right)^{4}\left(\frac{z}{5 \times 10^{4}}\right)^{3 / 2} \\
& \frac{1}{\tau_{\mathrm{db}}^{\text {hed }} H} \simeq 1.2 \times 10^{-3}\left(\frac{5 \mathrm{TeV}}{m_{\text {hed }}}\right)\left(\frac{z}{5 \times 10^{4}}\right)^{3 / 2}
\end{aligned}
$$

Here we have used the expansion rate in the radiation-dominated era, $H(z) \propto(1+z)^{2}$. For the CCDM model, the ratio of the interaction and expansion rate increases with time and is a constant in the matter dominated era. For the other two cases, the rate of interaction is greater than the expansion rate at early times.

\section{Evolution of coupled dark matter-baryon density and velocity pertur- bations}

The interaction of all the proposed dark matter candidates occurs via momentum transfer with baryons. The dark matter-photon coupling is sub-dominant to the electron-photon coupling in all the cases we consider and therefore we neglect it. The main impact of the additional interaction is to alter the evolution of density $(\delta)$ and velocity perturbations $(\theta)$ of the coupled photon-baryons fluid along with the interacting component of the dark matter. The relevant equations in the Newtonian/Conformal gauge are (for details see e.g. [50]):

$$
\begin{aligned}
& \dot{\delta_{c}}=-\theta_{c}+3 \dot{\phi} \\
& \dot{\theta_{c}}=-\frac{\dot{a}}{a} \theta_{c}+k^{2} \psi+\frac{a}{\tau_{\mathrm{db}}}\left(\theta_{b}-\theta_{c}\right) \\
& \dot{\delta_{b}}=-\theta_{b}+3 \dot{\phi} \\
& \dot{\theta_{b}}=-\frac{\dot{a}}{a} \theta_{b}+k^{2} \psi+\frac{a R}{\tau_{\mathrm{e} \gamma}}\left(\theta_{\gamma}-\theta_{b}\right)+\frac{a}{\tau_{\mathrm{bd}}}\left(\theta_{c}-\theta_{b}\right) \\
& \dot{\delta_{\gamma}}=-\frac{4}{3} \theta_{\gamma}+4 \dot{\phi} \\
& \dot{\theta_{\gamma}}=k^{2}\left(\frac{\delta_{\gamma}}{4}-\sigma_{\gamma}\right)+k^{2} \psi+\frac{a}{\tau_{\mathrm{e} \gamma}}\left(\theta_{b}-\theta_{\gamma}\right)
\end{aligned}
$$

Here the dot corresponds to the derivative with respect to the conformal time, $d \eta=d t / a$. $\tau_{\mathrm{e} \gamma}=1 /\left(n_{e} \sigma_{\mathrm{T}} c\right)$ determines the interaction time scale between baryons and photons while $R=$ $4 \rho_{\gamma} /\left(3 \rho_{b}\right)$ denotes the relative inertia of baryons and photons in the scattering process and this factor ensures: (a) the entire baryonic fluid shares the momentum exchange between photons and electrons and (b) this scattering process is momentum conserving. $\tau_{\mathrm{bd}}$ and $\tau_{\mathrm{db}}$ are the interaction time scales between baryons and dark matter. Here it is again implicitly assumed the momentum exchange between the two particles is shared by the entire baryonic fluid. These time scales are derived and discussed in section 2.3. As shown in section $2.3, \tau_{\text {bd }}^{-1}=$ $\rho_{\mathrm{dm}} \tau_{\mathrm{db}}^{-1} / \rho_{b}$. Here the mass density of interacting component of dark matter, $\rho_{\mathrm{dm}}=m_{\mathrm{dm}} n_{\mathrm{dm}}$, with $m_{\mathrm{dm}}$ and $n_{\mathrm{dm}}$ being the mass and the number density interacting dark matter particles, respectively. In our study we assume all the species to be at the same temperature (see 
later sections for details). $\psi$ and $\phi$ are cosmological potentials in the Newtonian/conformal gauge, $\sigma_{\gamma}$ is the second moment of the photon distribution, and all the other variables have their usual definitions (for details see [50]). The rest of the equations of the coupled multicomponent fluid along with Einstein's equations remain the same. We solve these equations by modifying the publicly-available code CLASS [17].

The main impact of the models considered here is captured by the last terms in Eqs. (3.2) and (3.4). To solve these equations along with Eq. (3.6), we use the tight coupling approximation whenever the relevant time scales are much shorter than the expansion time scale; the details of this approximation are discussed in the next section. When the time scales of interaction between baryons and dark matter exceed the expansion time scale, Eq. (3.2) can be integrated directly and Eq. (3.4) can be merged with Eq. (3.6) in the usual tight-coupling expansion in $\tau_{\mathrm{e} \gamma}$.

\subsection{Dynamics of baryon-photon-dark matter fluid: tight-coupling approxima- tion}

We draw upon Eqs. (3.2)-(3.6) in this section. We first briefly discuss the usual case in which there is no coupling between dark matter and baryons. In this case, the dynamics of the coupled baryon-photon fluid is determined by a two time scales $\tau_{\mathrm{e} \gamma}$ and $\tau_{\mathrm{e} \gamma} / R ; R=$ $4 \rho_{\gamma} /\left(3 \rho_{b}\right) \gg 1$ in the early universe as it scales as $1 / a$. Both these time scales are much shorter than the expansion time scale before the recombination sets in close to $z \simeq 1100$, which makes it difficult to numerically solve the coupled evolution of the baryon-photon fluid in the early universe. However, for the scales of interest, the photon-baryon fluid can be treated using tight-coupling approximation for $z \gg 1000$. In this approximation, the two fluids oscillate with a common sound velocity: $c_{s} \simeq c \sqrt{R /(3(1+R))}$. To take into account both the common oscillation of the fluid and Silk damping owing to the finite mean free path of the photon, one needs to solve the coupled photon-baryon equations up to second order in $\tau_{\mathrm{e} \gamma}$. The exact equations can be numerically solved when $\tau_{e \gamma} \simeq H^{-1}$. This approach is adopted in all the CMB codes that numerically solve the coupled baryon-photon evolution (e.g. [50]).

The interaction between baryons and dark matter introduces additional complications. For two of the models we consider, DD and $\mathrm{HeD}$, the tight-coupling approximation also applies to the coupled baryon-dark matter system in the early universe. In the early universe, all the four time scales $-\tau_{e \gamma}, \tau_{e \gamma} / R, \tau_{\mathrm{db}}$ and $\tau_{\mathrm{bd}}$-are shorter than the expansion time scales, and the baryon-photon-dark matter fluids can be treated as tightly coupled.

In this case, $\theta_{b}-\theta_{\gamma}$ in Eq. (3.6) and $\theta_{b}-\theta_{c}$ in Eq. (3.2) can be expanded in $\tau_{\mathrm{e} \gamma}$ and $\tau_{\mathrm{db}}$, respectively, and substituted in Eq. (3.4). This gives us:

$$
\left(1+R+R^{\prime}\right) \dot{\theta}_{b}+\frac{\dot{a}}{a}\left(\theta_{b}+R^{\prime} \theta_{c}\right)-k^{2} R\left(\frac{\delta_{\gamma}}{4}-\sigma_{\gamma}\right)+R\left(\dot{\theta}_{\gamma}-\dot{\theta}_{b}\right)+R^{\prime}\left(\dot{\theta}_{c}-\dot{\theta}_{b}\right)=\left(1+R+R^{\prime}\right) k^{2} \psi
$$

Here $R^{\prime}=\tau_{\mathrm{db}} / \tau_{\mathrm{bd}}=\rho_{\mathrm{dm}} / \rho_{b}$. Eq. (3.7) is exact. To obtain the equation which is correct to first order in $\tau_{e \gamma}$ and $\tau_{\mathrm{db}}$, we put $\sigma_{\gamma}=0$ and $\theta_{b}=\theta_{\gamma}=\theta_{c}$. In the absence of dark matterbaryon coupling $\dot{\theta}_{\gamma}-\dot{\theta}_{b}$ in Eq. (3.7) needs to be expanded to first order in $\tau_{e \gamma}$ to correctly account for photon diffusion damping (Silk damping). We note that this also suffices in our case as the diffusion damping owing to baryon-dark matter coupling impacts much smaller 
scales ${ }^{7}$; the evolution of $\theta_{\gamma}$ can be obtained after eliminating $\theta_{\gamma}-\theta_{b}$ in Eqs. (3.6) and (3.4) (e.g. Equations 70, 74, and 75 of [50]). Similarly, the suitable equation for the evolution of $\theta_{c}$ can be obtained from Eqs. (3.2) and (3.4). Eq. (3.7) allows us to compute the approximate sound speed in the coupled baryon-photon-dark matter fluid: $c_{s}^{2} \simeq R /\left(3\left(1+R+R^{\prime}\right)\right)$.

The DD and HeD models we consider in this paper correspond to cases in which the decoupling of dark matter and baryons occurs in the redshift range $z>10^{4}-10^{6}$. If all the cold dark matter is in the form of these atoms, which is allowed (section 4 ), $R^{\prime}=\rho_{c} / \rho_{b} \simeq 5.5$. For comparison, $R \simeq 100$ at $z \simeq 10^{5}$. This means $R^{\prime} \ll R$ for a majority of the cases we consider.

In the CCDM model, the baryon-dark matter coupling is weak at early times. This allows us to solve the dynamics of dark matter and baryons-photon fluid separately at early time. When this coupling becomes large (at $z \leq 5000$ for the models we study), the system of equations can be solved in the tight-coupling approximation. For all the models that are compatible with cosmological observables, the fraction of CCDM dark matter, $f_{\text {ccdm }}$, is a few percent. This implies $R^{\prime} \ll R$ for this case also.

For $R^{\prime} \ll R$ and $R^{\prime} \ll 1$, it follows from Eq. (3.7) that the dark matter-baryon coupling has negligible impact on the dynamics of the photon-baryons fluid while the dynamics of the interacting component of the dark matter is significantly affected by the coupling.

\subsection{Matter power spectrum and CMB anisotropies}

The tight coupling approximation can be used to gauge the impact of the additional coupling on the matter power spectrum and CMB anisotropies.

In the usual $\Lambda \mathrm{CDM}$ case, perturbations (in the Newtonian gauge) in all the matter components are constant at superhorizon scales. At sub-horizon scales, the cold dark matter perturbations grow logarithmically in the radiation-dominated era and as $\eta^{2}$ in the matterdominated era. The photons and baryons are tightly coupled before recombination era and their coupled perturbations at sub-horizon scales oscillate with a constant amplitude (e.g. [30]).

In the presence of additional dark matter-baryon coupling, a fraction (or all) of dark matter could behave as baryon-photon fluid. This means that the perturbations in this dark matter component cannot grow either logarithmically or as $\eta^{2}$ before the era of recombination. This causes a suppression in the matter power spectrum at scales that are sub-horizon when the coupling is strong. For the DD and HeD models, the coupling is strong at only early times and therefore the smaller scales are affected. For the CCDM case, the coupling is strong at latter times which impacts scales that enter the horizon around the time of recombination. This is clearly seen in the Figures 1 and 2.

The CMB anisotropies are sensitive to perturbations close to the epoch of recombination, $\eta \simeq \eta_{r}$. The angular scale of the observed anisotropies $\ell$ correspond approximately to the Fourier mode of perturbations $k$ as $\ell \simeq k \eta_{0}$, where $\eta_{0} \simeq 14400 \mathrm{Mpc}$ correspond to the conformal time at the present. As Planck can measure anisotropies for angular scales $\ell<2500$, the CMB anisotropies carry information of Fourier modes, $k<0.2 \mathrm{Mpc}^{-1}$. The primary source term for the CMB anisotropies is (e.g. [52, 53]):

$$
\mathcal{S}\left(\eta_{r}, k\right) \simeq \phi\left(\eta_{r}, k\right)+\frac{\delta_{\gamma}\left(\eta_{r}, k\right)}{4}
$$

\footnotetext{
${ }^{7}$ The typical scales impacted by Silk damping owing to photon diffusion, $\lambda_{\mathrm{b} \gamma}(t) \simeq c \sqrt{\tau_{\mathrm{e} \gamma} H}$ (see e.g. [30]) while the corresponding scale for diffusion owing to dark matter-baryon coupling is $\lambda_{\mathrm{bd}}(t) \simeq v \sqrt{\tau_{\mathrm{e} \gamma} H}$. For all the cases we discuss here $\lambda_{\mathrm{b} \gamma} \gg \lambda_{\mathrm{bd}}$.
} 

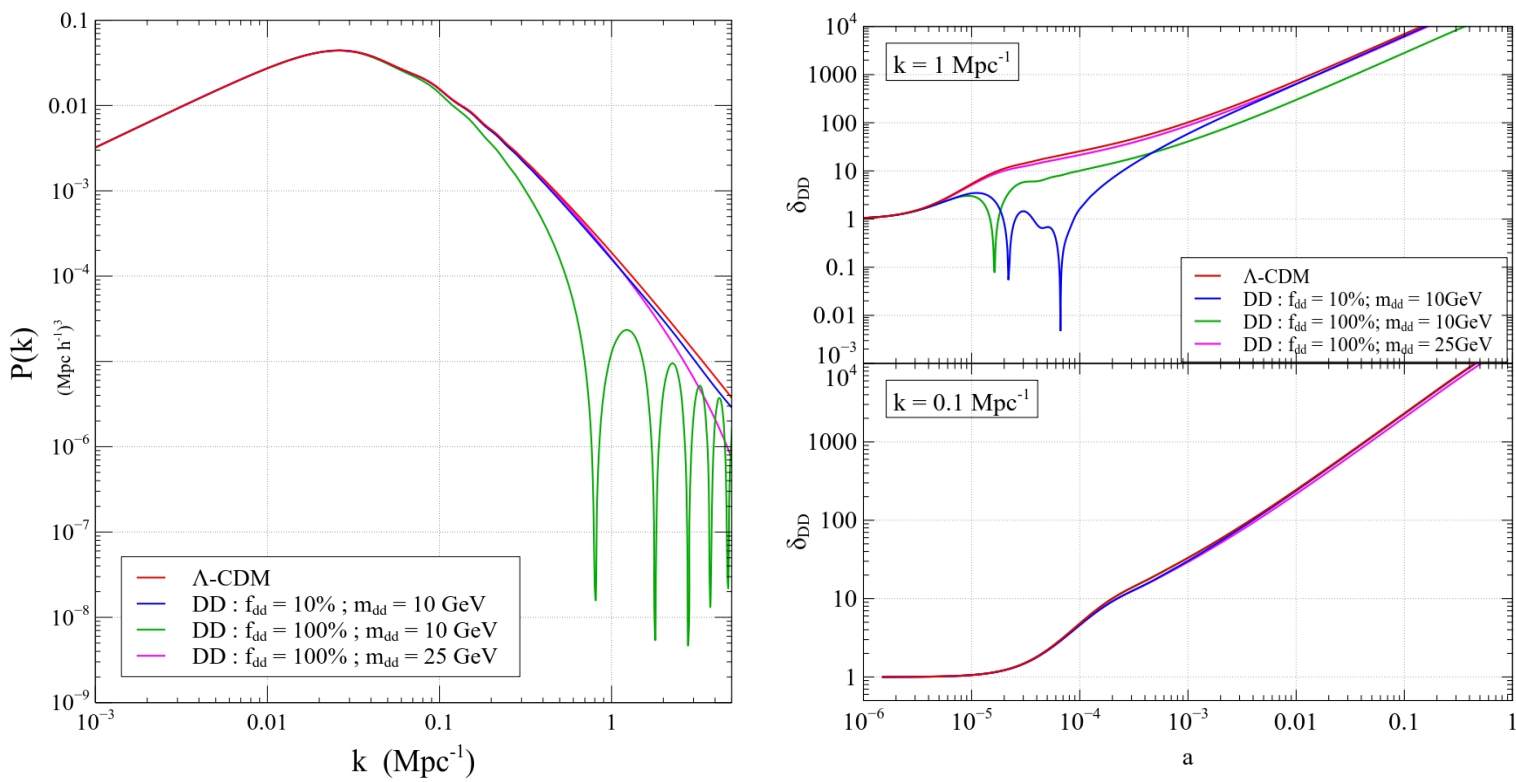

Figure 1. Left panel: The matter power spectra $P(k)(z=1000)$ are shown for many parameters of the DD model, along with the results of the usual $\Lambda$ CDM model. Right Panel: The time evolution of $\delta_{\mathrm{DD}}$ is displayed for two Fourier modes, $k=0.1 \mathrm{Mpc}^{-1}$ and $k=1 \mathrm{Mpc}^{-1}$.
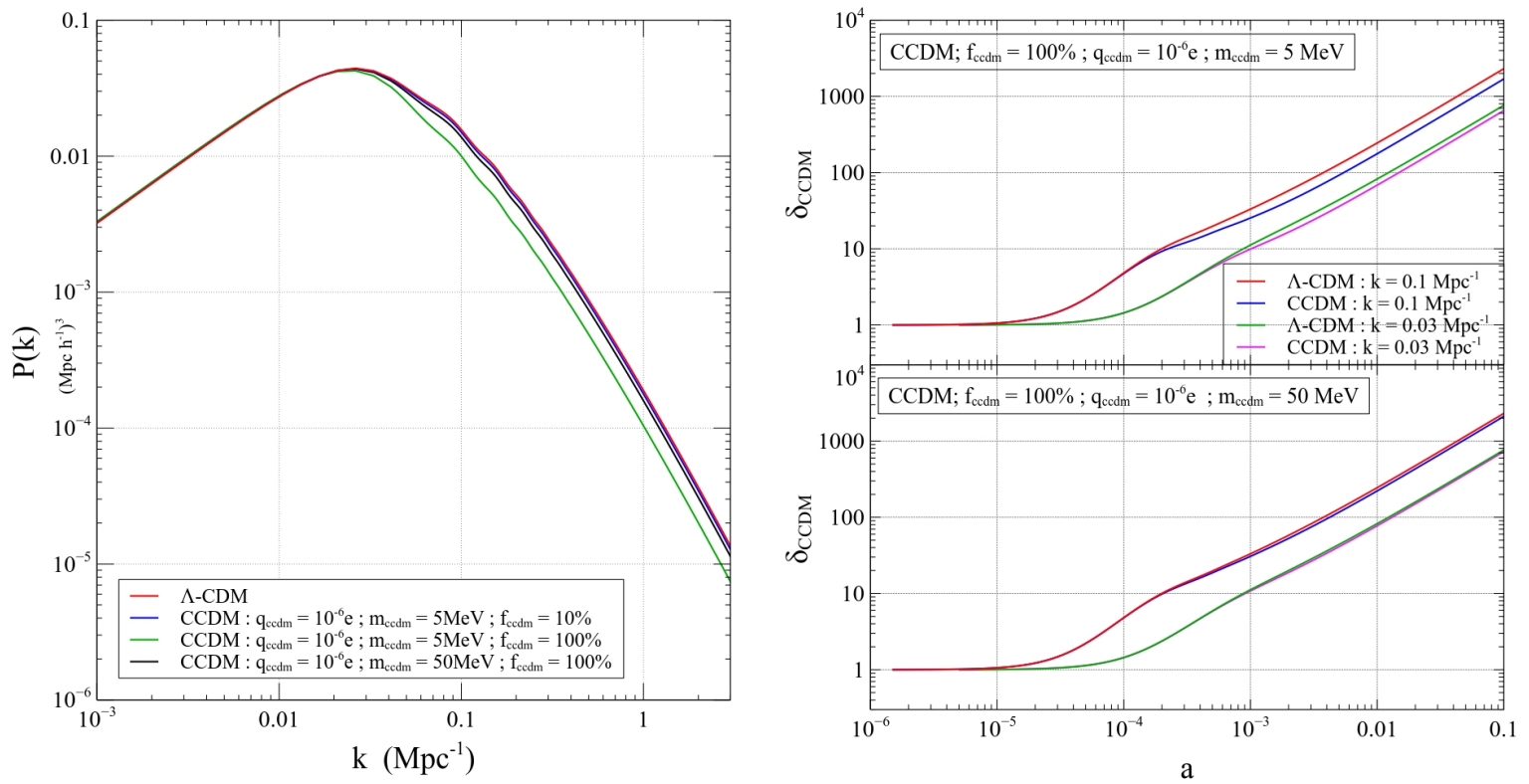

Figure 2. Left panel: The matter power spectra $P(k)$ are shown for several CCDM models at $z=1000$. The $\Lambda \mathrm{CDM}$ matter power spectrum is also shown for comparison. Right Panel: The time evolution of density contrast of the interacting component of the dark matter $\delta_{\mathrm{CCDM}}$ is displayed for two Fourier modes, $k=0.1 \mathrm{Mpc}^{-1}$ and $k=0.03 \mathrm{Mpc}^{-1}$.

At large scales, $k \eta_{r} \ll 1$, the source term , $\mathcal{S}\left(\eta_{r}, k\right)=\phi\left(\eta_{r}, k\right) / 3$ yields the well-known SachsWolfe effect. These scales are not affected by the baryon-dark matter coupling. The source term at smaller scales, $k \eta_{r}>1$, is difficult to compute analytically. Approximate expressions 
have been used in the literature to capture the essential physics at these scales. We use the analysis presented by Mukhanov [52] to underline the impact of the additional coupling on CMB anisotropies. Following [52], the source term for $k \eta_{r}>$ is:

$$
\mathcal{S}\left(\eta_{r}, k\right) \simeq\left[\frac{\log \left(b_{1} k \eta_{\mathrm{eq}}\right)}{\left(b_{2} k \eta_{\mathrm{eq}}\right)^{2}}\left(1-\frac{1}{3 c_{s}^{2}}\right)+2 \sqrt{c_{s}} \cos \left(k \int_{0}^{\eta_{r}} c_{s} d \eta\right) \exp \left(-k / k_{D}\right)^{2}\right] \phi\left(\eta_{i}, k\right)
$$

Here $\eta_{i}$ corresponds to an initial time at which $k \eta_{i} \ll 1$. $\eta_{\text {eq }}$ is the conformal time at matterradiation equality and $c_{s} \simeq \sqrt{R /(3+3 R)}$ is the sound velocity of the coupled baryon-photon fluid at the recombination era. $k_{D}^{2} \simeq 1 /\left(\eta \tau_{e \gamma}\right)$ gives the Silk damping scale. $b_{1}$ and $b_{2}$ are constants whose values depend on $k$. Eq. (3.9) allows us to assess the impact of baryonphoton coupling on the observed CMB anisotropies. First, as already discussed above, the Silk damping scale $k_{D}$ is not affected by this coupling.

In $\mathrm{DD}$ and $\mathrm{HeD}$ models, the decoupling occurs at early times and therefore quantities such as $c_{s}$ whose value only close to the recombination era contribute significantly to CMB anisotropies are not affected. The main impact comes from the first term on the RHS of Eq. (3.9) which arises from the time evolution of the gravitational potential $\phi(\eta, k) . \phi(\eta, k)$ is constant at superhorizon scales in the radiation-dominated era and is constant at all scales in the matter-dominated era. Neglecting the impact of cold dark matter perturbations, the potential decays as $1 / \eta^{2}$ for scales that enter the horizon during the radiation-dominated era. The perturbations of the cold dark matter grow logarithmically for sub-horizon scales during the radiation-dominated era. And when this effect is taken into account, the potential falls slower than $1 / \eta^{2}$; this is the origin of the log term in Eq. (3.9). For DD and HeD models, the cold dark matter perturbation cannot grow when the baryon-dark matter coupling is strong, which diminishes the value of $\log$ term in Eq. (3.9). As the values of $b_{1}$ and $b_{2}$ are strong functions of $k$ (e.g. $[30,52,53]$ ), we do not try to estimate it analytically here but only present numerical results for the matter power spectrum (Figures 1 and 2).

In the CCDM case, the baryon-dark matter coupling becomes stronger at later time and its main impact occurs close to the time of decoupling. CMB data strongly constrains the angle subtended on by the sound horizon at the epoch of recombination, $\theta_{\star}=r_{s} / D_{A}$, where $r_{s} \simeq \eta_{r} \sqrt{R /(3(1+R))}$ and $D_{A}$ is the angular diameter distance to the epoch of recombination. Planck data yields $\theta_{\star}=0.59643 \pm 0.00026$ (in degrees) [56]. In the usual case, $R=4 \rho_{\gamma} /\left(3 \rho_{b}\right)$ and $R \simeq 1.2$ at $z \simeq 1000$. If all the dark matter is in the form of CCDM particles, and these particles are strongly coupled to baryons at recombination, $r_{s} \simeq \eta \sqrt{R /\left(3\left(1+R+R^{\prime}\right)\right)}$, which is radically different from the usual value as $R^{\prime} \simeq 6$. This is readily ruled out by the CMB data. Therefore, we only consider models for which $R^{\prime} \ll 1$, or the interacting dark matter must be a small fraction of baryons. In such cases, the term corresponding the potential evolution in Eq. (3.9) is minimally affected and the main impact of CCDM models on CMB anisotropies is owing to the change in $c_{s}$ in the second term on the RHS because of non-zero $R^{\prime}$.

\subsubsection{Numerical implimentation in CLASS}

It follows from the discussion above that for the $\mathrm{DD} / \mathrm{HeD}$ cases, the dark matter is tightly coupled at high redshifts and then decouples when the interaction rate between the dark matter and baryons falls below the expansion rate. On the other hand, the coupling becomes stronger at later time in CCDM case.

For numerical stability, we switch from the tight-coupling approximation to the exact equations when the dominant interaction rate is 100 times the expansion rate for the CCDM 
model. As noted above, the CCDM and baryon cannot be tightly coupled at the last scattering surface for viable cosmological models, if the fraction of CCDM is substantial. This allows us to use the exact equations for a majority of the cases we study. For the DD/HeD cases we make the switch when the interaction rate is 20 times the expansion rate. This allows us to evolve wavenumbers $k \lesssim 10 \mathrm{Mpc}^{-1}$ without glitches. We note that this prescription is similar to and in addition to the usual case in which the photon-baryon plasma makes transition from tightly coupled to photon decoupling close to the epoch of recombination.

The initial conditions for the CCDM case are the same as in the usual case because the milli-charged dark matter particle is upcoupled from the baryons at early times. In the other two cases, a fraction of the dark matter is tightly coupled to baryons at early times so the initial condition for bulk velocity of this component is derived from the tight-coupling relation: $\theta_{c}=\theta_{b}$. The other initial conditions remain the same.

In section 2 we discuss the formation of $\mathrm{DD}$ and HeD atoms. The formation redshift of these atoms is before the initial conditions are set in CLASS: radiation domination era with scales of interest $\left(k \lesssim 10 \mathrm{Mpc}^{-1}\right)$ well outside the horizon. However, we note that the initial conditions are immune to whether the dark matter is in free (ionized) form or in atomic form because the interaction rate of both these components far exceeds the expansion rate when initial conditions are set. All the components that are coupled to the baryonphoton plasma share a common bulk velocity with it, as follows from the discussion on tight-coupling approximation in the foregoing. This situation is akin to how different stages of ionization of helium nuclei are incorporated in numerical codes in the usual $\Lambda$ CDM casethe neutral helium is treated the same way as ionized helium because the interaction rates of both components with electrons/protons far exceeds the expansion rate.

\section{Matter power spectrum}

The last section provides a general framework to study cosmological implications of additional baryon-dark matter interaction. In this section we focus on specific models and their matter power spectra. We build on the discussion in Section 3.1 and provide numerical results.

It follows from Eqs. (3.2) and (3.4) that all the models tend towards the standard $\Lambda \mathrm{CDM}$ case when interaction rates fall below the expansion rates at redshifts of interest: $\tau_{i j} \gg H^{-1}(z)$. The redshifts of interest can be determined from the following argument. When a scale is outside the horizon, $k \eta<1$, both the baryonic and dark matter perturbations evolve in the same way (for details e.g. [50]). If the baryons and dark matter are coupled during this phase but get decoupled before the scale enters the horizon, the impact of the additional coupling is negligible at that scale, i.e. large scales $k<0.02 \mathrm{Mpc}^{-1}$ that enter the horizon after the epoch of recombination fall in this category. CMB anisotropies and galaxy clustering data are sensitive to scales $k<0.2 \mathrm{Mpc}^{-1}$. If these scales enter the horizon when the baryon-dark matter coupling is strong then we expect observable signatures on the CMBR anisotropies and the matter power spectrum. The redshifts of interest from the point of view of observable signatures are $10^{3}<z<10^{5}$, which corresponds to the time between the epoch of recombination and roughly the epoch at which the mode $k \simeq 0.3 \mathrm{Mpc}^{-1}$ enters the horizon.

CCDM model: Eqs. (2.1) and (2.16) show that the CCDM particles and baryons get coupled at late times. To have a significant impact on cosmological observables this coupling must be strong before the recombination era, which occurs when either of the two time scales, $\tau_{\mathrm{db}}$ and $\tau_{\mathrm{bd}}$, become comparable to the expansion time scale. In the two panels of Figure 2, 
we show the matter power spectra of the CCDM model and display the time evolution of density perturbations of the interacting component of the dark matter, $\delta_{\mathrm{CCDM}}$, for a few Fourier modes to glean the important features introduced by CCDM models.

It follows from the discussion in section 3.1 that the fraction of dark matter in the form of CCDM, $f_{\mathrm{CCDM}} \ll 1$, if the baryons and CCDM particles are tightly coupled at $z \simeq 1000$. In Figure 2, we show a few cases where the coupling is not strong enough to cause coupled oscillation of the baryon-photon-dark matter fluid but strong enough to significantly alter the matter power spectrum.

As argued in section 3.1, we expect the matter power to diminish in the presence of CCDM as the baryon-dark matter coupling prevents the growth of CDM perturbations. As the coupling becomes stronger with time, all the scales that enter the horizon before the recombination era are affected in this case. We notice these features in Figure 2. The main impact on $\mathrm{CMB}$ anisotropies in this case occurs owing to the change the sound velocity of the coupled baryon-photon fluid at $z \simeq 1000$, as discussed in section 3.1.

DD atom: The physics of the formation of this atom and its interaction with baryons are discussed in detail in section 2. Eq. (2.17) shows that this atom is coupled to baryons at early times and the coupling becomes weaker with time. The scattering cross section of DD atoms off baryons scales as $1 / m_{\mathrm{dd}}^{3}$ (Eq. (2.12)) and therefore the redshift at which the decoupling occurs is a very sensitive function of the dark matter mass. In Figure 1 we show the matter power spectrum for several models in which the DD atom could constitute either a part or all of the cold dark matter. In the right panel of Figure 1, we also show the time evolution of $\delta_{\mathrm{DD}}$, the density contrast of the interacting component of the dark matter.

The scales that are most affected by dark matter-baryon interaction are those that are inside the horizon when this coupling is still strong. For instance, if the decoupling occurs at $z \simeq 10^{5}$, perturbations at scales $k>0.3 \mathrm{Mpc}^{-1}$ are affected by this coupling.

From the discussion in section 3.1, we can assess the impact of this additional coupling on the evolution of different components in the tight-coupling approximation. The DD case allows for all the cold dark matter to be in the form of interacting dark matter or $R^{\prime}=$ $\rho_{\text {dd }} / \rho_{b} \simeq 5$.5. For $R \gg R^{\prime}$ and $R^{\prime} \ll 1$, the coupled baryon-photon fluid is negligibly affected by this new coupling but perturbations of the interacting component of the dark matter are driven by the oscillations of the baryon-photon fluid. When $R^{\prime} \gtrsim 1$, the baryon-photon perturbations are also significantly affected by this coupling. If the coupling is strong, the three fluids oscillate together with a common sound velocity, $c_{s} \simeq \sqrt{\left(R /\left(3\left(1+R+R^{\prime}\right)\right)\right.}$, which differs markedly from the sound velocity of the baryon-photon fluid.

This discussion allows us to understand the features seen in Figure 1: (a) the matter power spectrum approaches the $\Lambda \mathrm{CDM}$ models as $m_{\mathrm{dd}}$ is increased and the $f_{\mathrm{DD}}$ is lowered, (b) the interacting part of the dark matter behave as the baryon-photon fluid for scales that enter the horizon when the coupling is strong. This explains the oscillations seen in the matter power spectrum for the model: $m_{\mathrm{dd}}=10 \mathrm{Gev}$ and $f_{\mathrm{DD}}=1$. This behaviour is also evident in the time evolution of density perturbations (right panel), e.g. for $k=1 \mathrm{Mpc}^{-1}$. For the models displayed in Figure 1, the decoupling occurs for $z>10^{5}$.

HeD atom: Like the DD case, the coupling between baryons and HeD atoms is stronger at high redshifts (Eq. (2.18)). Therefore, the physics of this model is similar to the DD case with a few notable differences. Unlike the DD case, the dark matter-baryon scattering cross section does not depend on the mass of the dark matter particle (Eq. (2.12)). The only condition to ensure the formation and stability of this atom is that the dark matter particle be much heavier than the helium nucleus. Therefore, unlike CCDM and DD models, it is 

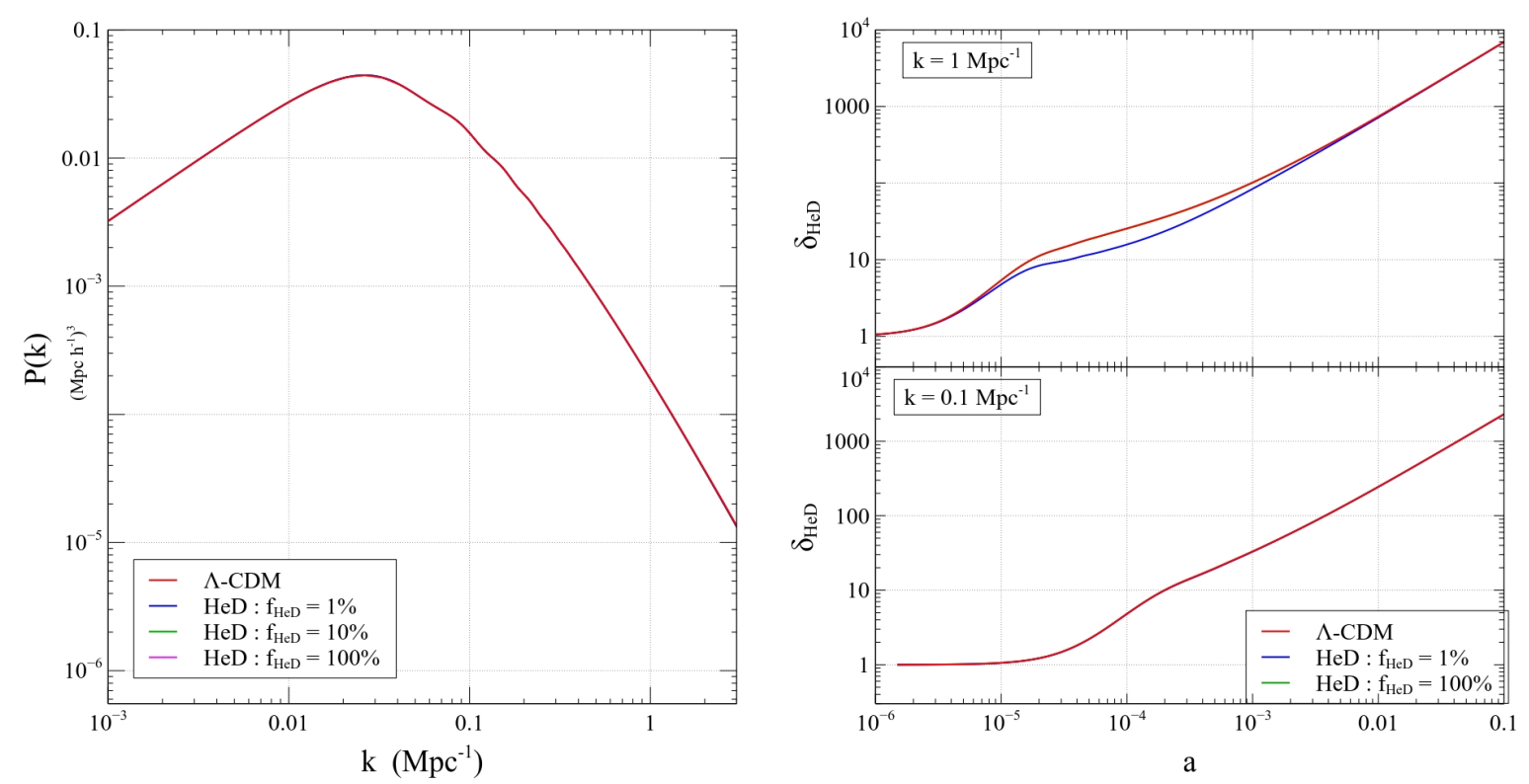

Figure 3. Left panel: The matter power spectra $P(k)$ are shown for HeD models for different values of $f_{\mathrm{HeD}}$ at $z=1000$, along with the usual $\Lambda \mathrm{CDM}$ model. Right Panel: The time evolution of $\delta_{\mathrm{HeD}}$ is displayed for two Fourier modes, $k=0.1 \mathrm{Mpc}^{-1}$ and $k=1 \mathrm{Mpc}^{-1}$.

harder to tune the parameters of this model to seek agreement with observations and this model is a more robust representative of a paradigm that admits electromagnetic interaction between dark matter and baryons.

An additional requirement in this model is that only a small fraction of ${ }^{4} \mathrm{He}$ be captured by dark matter particles to form $\mathrm{HeD}$ atoms. From the current astronomical and CMB data the primordial abundance of ${ }^{4} \mathrm{He}$ could be constrained to better than one percent (e.g. $[42,56]$ and references therein ${ }^{8}$ ). Therefore, we consider models in which only $1 \%$ of helium nuclei form atoms with dark matter. This gives us: $n_{\text {hed }} \simeq 0.0008 n_{b}$ and $m_{\mathrm{dm}} \simeq m_{p} f_{\mathrm{HeD}} \rho_{\mathrm{dm}} n_{b} /\left(\rho_{b} n_{\text {hed }}\right)$, where $f_{\mathrm{HeD}}$ is the fraction of dark matter in the form of $\mathrm{HeD}$ atoms. Using the Planck best-fit values of $\rho_{\mathrm{dm}}$ and $\rho_{b}$, we get, $m_{\mathrm{hed}} \simeq 7000 f_{\mathrm{HeD}} m_{p}$. Owing to the large mass of the particle that recombines with helium nucleus to form the HeD atom, the interaction rate between baryons and $\mathrm{HeD}$ atoms is much smaller (Eq. (2.15)). For all the models we consider for different values of $f_{\mathrm{HeD}}$, the matter power spectrum is an excellent agreement with the $\Lambda \mathrm{CDM}$ model for $k \leq 1 \mathrm{Mpc}^{-1}$. In Figure 3 we show the matter power spectra and the time evolution of two Fourier modes for a few cases.

\subsection{CMB anisotropies}

It follows from the discussion in section 3.1 that for the $\mathrm{DD}$ and $\mathrm{HeD}$ cases the impact of the additional baryon-dark matter coupling can be captured by the change in the matter power spectrum at scales that are inside the horizon during strong baryon-dark matter coupling. For the milli-charged particle, the change in the velocity of sound close to the recombination provides an additional discriminator.

\footnotetext{
${ }^{8} \mathrm{CMB}$ anisotropies have a bearing on the Helium abundance by constraining the number of electrons captured by Helium nuclei close to the epoch of recombination. In the HeD model, the charge neutrality of the universe requires us to have fewer electrons as compared to the usual model. The CMB anisotropies are sensitive to this deficit.
} 

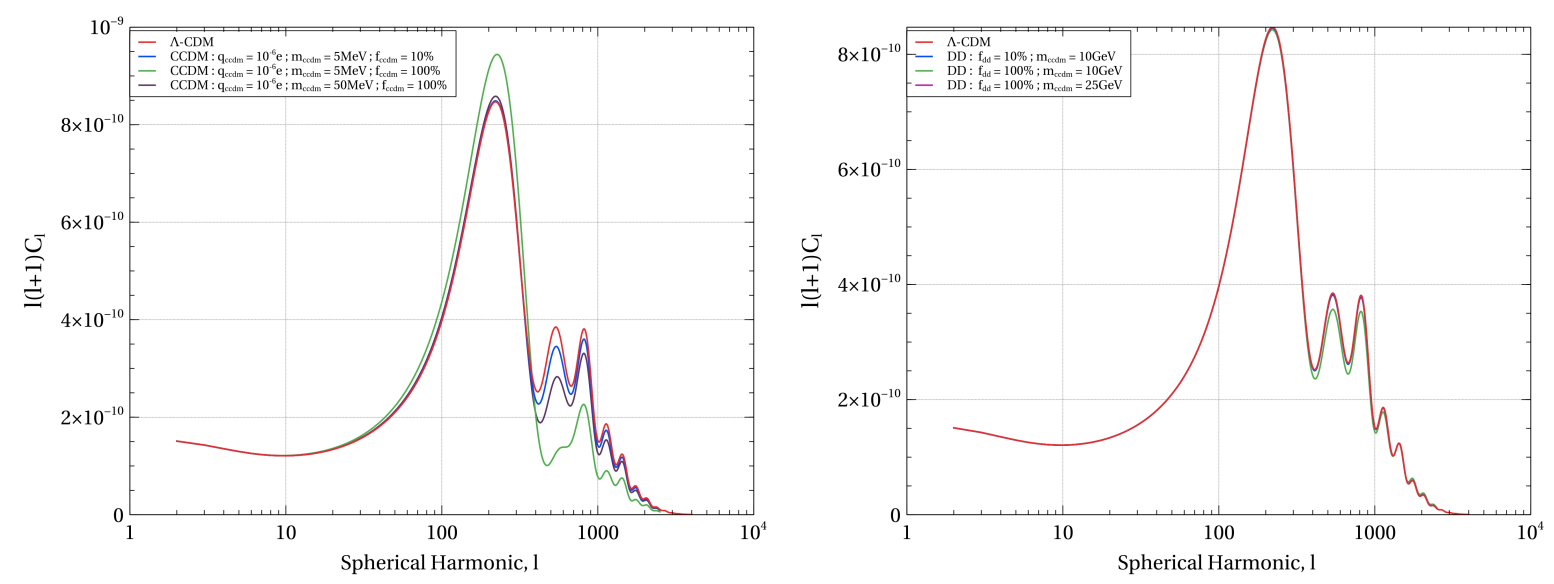

Figure 4. The angular power spectra of CMB temperature anisotropy are shown for a few CCDM (DD) models along with the $\Lambda$ CDM model.

The angular scale of CMB anisotropies $(l)$ can be related to scale of matter perturbations $(k)$ at last scattering surface using the approximation: $l \simeq k c \eta_{0}$, where $\eta_{0}=\int d t / a(t)$ is the conformal time at the present with $c \eta_{0} \simeq 14000 \mathrm{Mpc}$. In Figure 4, we show the CMB temperature angular power spectrum for a subset of models. As anticipated in the previous section, the main impact of DD models is on large $l$ while the CCDM also affects large angular scales $l \simeq 200$, which corresponds to wavenumbers that enter the horizon close to the epoch of recombination. We do not show the expected CMB angular power spectrum for the HeD model as, for permissible range of parameters, it is indistinguishable from the usual model, in line with discussion in the previous section.

\section{Comparison with data}

Our theoretical predictions can be compared to the existing CMB and galaxy clustering data. The current Planck data is sensitive to $k<0.2 \mathrm{Mpc}^{-1}[3]$ and the SDSS galaxy clustering data can also be compared to the prediction of linear theory for $k<0.1 \mathrm{Mpc}^{-1}$ (e.g. [16]). As these scales are comparable and the CMB data is less affected by the effects of non-linear clustering unlike the low-redshift SDSS data, we choose to compare our results against Planck 2018 CMB anisotropy data $[56]^{9}$. The following Planck likelihoods were used: high- $l$ temperature and polarization anisotropies $(l=30-2508$ for TT data and $l=30-1996$ for TE and EE data), low- $l$ temperature and polarization data $(l=2-29)$, and CMB lensing reconstruction. The models were analysed using the MCMC code MontePython [21].

Parameterization of models: (a) CCDM: The three free parameters of this model are: the charge of the dark matter particle, $q_{\mathrm{ccdm}}$, the mass of the dark matter particle, $m_{\mathrm{ccdm}}$, and the fraction of charged CDM, $f_{\mathrm{ccdm}}$. As discussed in section 3.1, if CCDM-baryon coupling becomes strong before the epoch of recombination, $f_{\text {ccdm }} \ll 1$. Here we explore models for which the coupling is not strong enough to cause coupled CCDM-baryon oscillations. It is important to underline that $\mathrm{CMB}$ anisotropies are extremely sensitive to behaviour of matter perturbations close to the epoch of recombination, (b) $D D$ : In this case, there are two free parameters, the mass of the dark matter particle, $m_{\mathrm{dd}}$, and the fraction of DD atoms, $f_{\mathrm{dd}}$.

\footnotetext{
${ }^{9}$ For details of the data products and likelihoods see https://wiki.cosmos.esa.int/planck-legacyarchive/index.php/CMB_spectrum_\%26_Likelihood_Code\#2018_Likelihood
} 


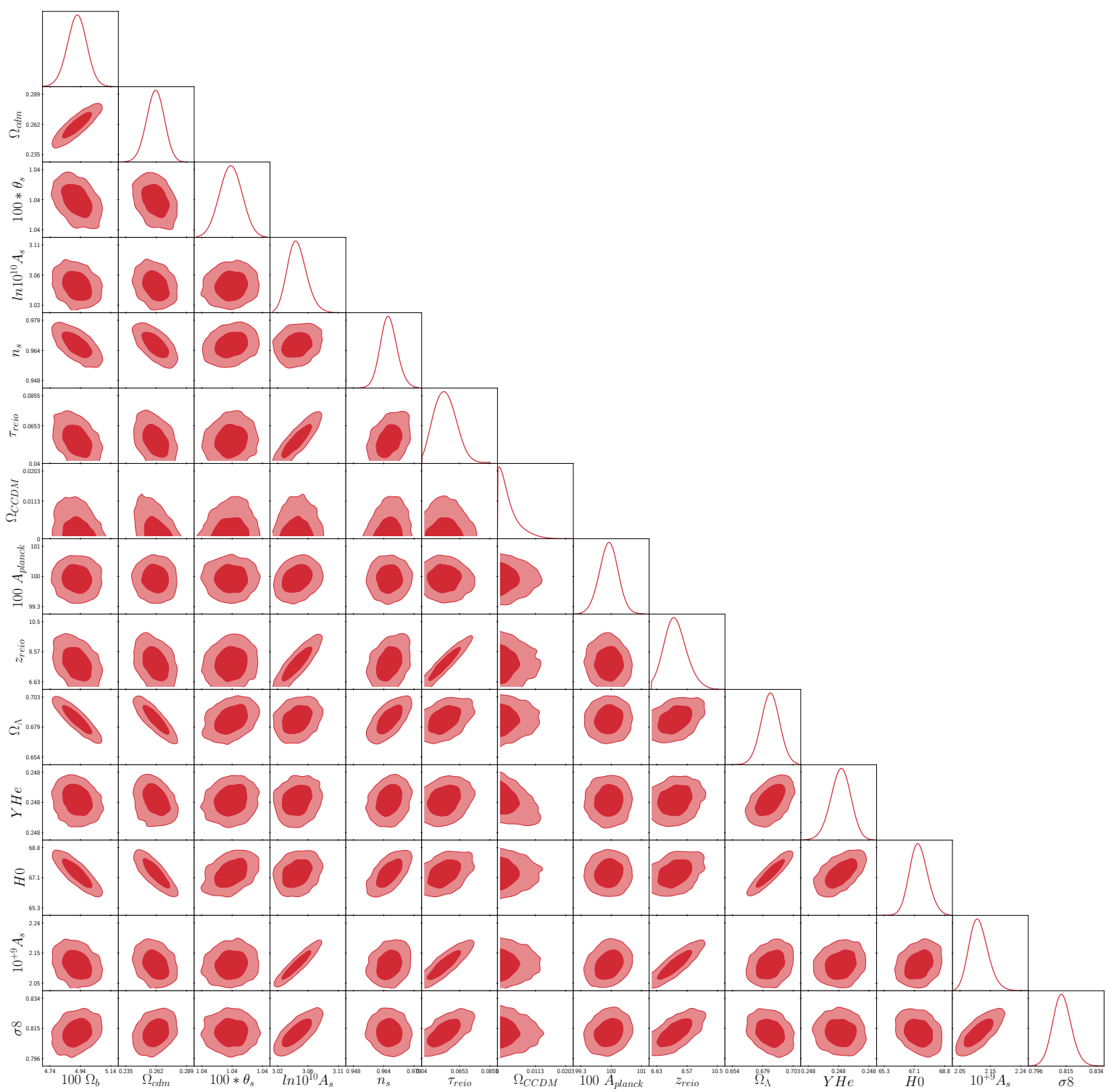

Figure 5. Triangle graph from the MCMC analysis for the CCDM model is shown for $m_{\mathrm{dm}}=50 \mathrm{MeV}$ and $q_{\mathrm{ccdm}}=10^{-6} \mathrm{e}$.

This model approaches the $\Lambda$ CDM model when $m_{\mathrm{dd}} \rightarrow \infty$ and/or $f_{\mathrm{dd}} \rightarrow 0$. For MCMC analysis, we fix the value of $m_{\mathrm{dd}}=10 \mathrm{GeV}$, which is close to the minimum mass allowed by charge-exchange stability (c) we do not carry out an MCMC analysis for the HeD case because, as noted above, all the models that are allowed by other astrophysical data are in excellent agreement with the $\Lambda \mathrm{CDM}$ model for $k<1 \mathrm{Mpc}^{-1}$ and therefore are not sensitive to the CMB data.

In Figures 5 and 6, we show the contour plots of cosmological parameters. The estimated best-fit parameters and their $2-\sigma$ errors are listed in Tables 1 and 2.

The comparison of our theoretical predictions with data shows that the current Planck data is consistent with no electromagnetically-interacting component of dark matter; $\Omega_{\mathrm{CCDM}}$ and $\Omega_{\mathrm{DD}}$ are consistent with zero. Figures 5 and 6 show that less than a few percent of the 


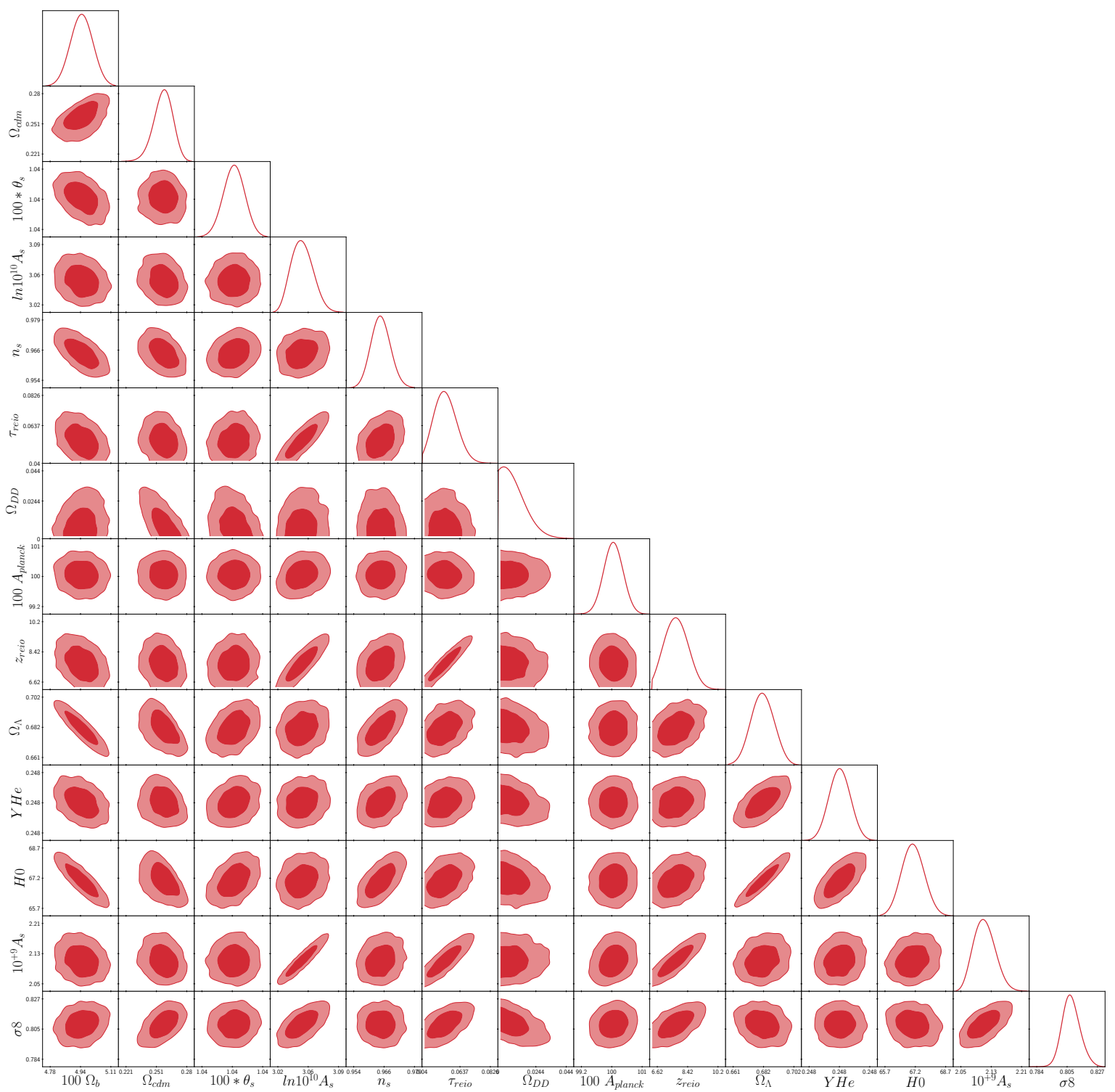

Figure 6. Triangle graph from the MCMC analysis for the DD model is shown for $m_{\mathrm{dd}}=10 \mathrm{GeV}$.

total non-relativistic dark matter (nearly $1 \%$ for the CCDM model and $4 \%$ for the DD model) could receive contribution from these components. Our results for the CCDM model are in general agreement with other cosmological analyses of the model (e.g. [47]).

\section{Conclusion and future prospects}

We have studied cosmological implications of three models of cold dark matter in which the dark matter interacts electromagnetically.

Our paper has three main aims:

- To study the formation, stability, and interaction of two classes of dark matter atoms, DD and HeD (Section 2). The third model, the extensively-studied milli-charged dark matter (CCDM), is used as a reference model for our study. 


\begin{tabular}{|l|c|c|c|c|}
\hline Param & best-fit & mean $\pm \sigma$ & $95 \%$ lower & $95 \%$ upper \\
\hline $100 \Omega_{b}$ & 4.978 & $4.916_{-0.064}^{+0.068}$ & 4.784 & 5.05 \\
$\Omega_{\mathrm{cdm}}$ & 0.2695 & $0.2612_{-0.0076}^{+0.0084}$ & 0.2453 & 0.2771 \\
$100 * \theta_{s}$ & 1.042 & $1.042_{-0.00028}^{+0.00032}$ & 1.041 & 1.042 \\
$\ln 10^{10} \mathrm{~A}_{\mathrm{s}}$ & 3.045 & $3.049_{-0.016}^{+0.014}$ & 3.021 & 3.078 \\
$n_{s}$ & 0.9625 & $0.9663_{-0.0044}^{+0.0045}$ & 0.9573 & 0.9754 \\
$\tau_{\text {reio }}$ & 0.05397 & $0.05576_{-0.0008}^{+0.0068}$ & 0.04148 & 0.07001 \\
$\Omega_{\mathrm{CCDM}}$ & 0.0004058 & $0.003395_{-0.0034}^{+0.0006}$ & $3.143 \times 10^{-7}$ & 0.009859 \\
$100 A_{\text {planck }}$ & 99.95 & $100.1_{-0.25}^{+0.26}$ & 99.53 & 100.6 \\
$z_{\text {reio }}$ & 7.686 & $7.84_{-0.78}^{+0.71}$ & 6.362 & 9.221 \\
$\Omega_{\Lambda}$ & 0.6788 & $0.6847_{-0.0078}^{+0.008}$ & 0.6694 & 0.7002 \\
$\mathrm{YHe}$ & 0.2478 & $0.2478_{-6.7 e-05}^{+7.3 e}$ & 0.2477 & 0.2479 \\
$H 0$ & 66.96 & $67.34_{-0.58}^{+0.55}$ & 66.24 & 68.44 \\
$10^{+9} A_{s}$ & 2.1 & $2.11_{-0.034}^{+0.029}$ & 2.052 & 2.172 \\
$\sigma_{8}$ & 0.8133 & $0.8124_{-0.0063}^{+0.006}$ & 0.7999 & 0.8245 \\
\hline
\end{tabular}

Table 1. The best fit parameters and $2 \sigma$ errors from MCMC analysis are displayed for the CCDM model, for the following parameters kept fixed: $m_{\mathrm{ccdm}}=50 \mathrm{MeV}$ and $q_{\mathrm{ccdm}}=10^{-6} \mathrm{e}$. The remaining parameter, the fraction of the milli-charged dark matter, $f_{\text {ccdm }}$ is varied, yielding the constraint on the density parameter $\Omega_{\mathrm{ccdm}} \equiv f_{\mathrm{ccdm}} \Omega_{\mathrm{dm}}$.

\begin{tabular}{|l|c|c|c|c|}
\hline Param & best-fit & mean $\pm \sigma$ & $95 \%$ lower & $95 \%$ upper \\
\hline $100 \Omega_{b}$ & 4.941 & $4.952_{-0.06}^{+0.064}$ & 4.828 & 5.074 \\
$\Omega_{\mathrm{cdm}}$ & 0.2623 & $0.2571_{-0.0087}^{+0.01}$ & 0.2377 & 0.2759 \\
$100 * \theta_{s}$ & 1.042 & $1.042_{-0.00029}^{+0.00032}$ & 1.041 & 1.042 \\
$\ln 10^{10} \mathrm{~A}_{\mathrm{s}}$ & 3.048 & $3.049_{-0.014}^{+0.013}$ & 3.023 & 3.076 \\
$n_{s}$ & 0.9649 & $0.965_{-0.0041}^{+0.0041}$ & 0.9568 & 0.9729 \\
$\tau_{\text {reio }}$ & 0.05517 & $0.0547_{-0.00078}^{+0.0062}$ & 0.04136 & 0.06814 \\
$\Omega_{\mathrm{DD}}$ & 0.004163 & $0.01086_{-0.011}^{+0.0028}$ & $4.962 \times 10^{-6}$ & 0.02694 \\
$100 A_{\text {planck }}$ & 100.1 & $100.1_{-0.24}^{+0.25}$ & 99.55 & 100.5 \\
$z_{\text {reio }}$ & 7.801 & $7.746_{-0.76}^{+0.65}$ & 6.379 & 9.105 \\
$\Omega_{\Lambda}$ & 0.6826 & $0.6811_{-0.0081}^{+0.0073}$ & 0.666 & 0.6967 \\
YHe & 0.2478 & $0.2478_{-6.6 e-05}^{+6.6 e-05}$ & 0.2477 & 0.2479 \\
$H 0$ & 67.18 & $67.09_{-0.57}^{+0.54}$ & 66 & 68.22 \\
$10^{+9} A_{s}$ & 2.108 & $2.11_{-0.031}^{+0.027}$ & 2.054 & 2.167 \\
$\sigma_{8}$ & 0.811 & $0.8087_{-0.0065}^{+0.007}$ & 0.7953 & 0.822 \\
\hline
\end{tabular}

Table 2. MCMC results for the DD model with $m_{\mathrm{dd}}=10 \mathrm{GeV} . \Omega_{\mathrm{dd}} \equiv f_{\mathrm{dd}} \Omega_{\mathrm{dm}}$, the energy density in the form of DD atoms, is constrained by the CMB data 
- To understand and compute, both analytically and numerically, the cosmological implications of these models (Section 3.1).

- To compare the predictions of these models with Planck CMB data.

One of the models (CCDM) involves coulomb interaction between charged particles and the dark matter. In this case, the interaction cross-section falls as $1 / v^{4}$ which causes stronger interaction at later times and therefore it impacts large scales which enter the horizon later. The other two models we study correspond to neutral atoms formed by the recombination of either two heavy singly charged particles (DD) or the recombination of a heavy doublycharged particle with Helium nucleus $(\mathrm{HeD})$. For the parameters of interest, the cross-section of interaction of these atoms with baryons is independent of velocity, which means the interaction is stronger at early times and the matter power spectrum at small scales is affected.

The DD and HeD models are qualitatively different from each other because the interaction cross-section strongly depends on the mass of the dark matter particle in the DD case while it is independent of the dark matter mass for the HeD atom. The HeD model is the most natural extension of the dark matter paradigm that admits a stable, electromagneticallyinteracting dark matter particle. The inference follows as the data rules out the other two compelling choices: stable dark matter particle of electronic charge or a neutral atom formed by the recombination of a proton and a dark matter particle. Our analysis shows that, for the range of other parameters allowed by astrophysical data, the HeD atom can constitute all the cold dark matter.

In the recent past, dark matter models with electromagnetic interactions have been invoked to explain the EDGES result [11, 19, 35, 47]. The EDGES result requires the baryons to be cooler at $z \simeq 20$ as compared to the usual model. One plausible explanation of this result is coloumbic interactions between milli-charged dark matter and baryons. It is of interest to investigate the cosmological implications of the other two models we study in the post-recombination universe. We note that models of atomic dark matter cannot explain the EDGES result as, unlike the CCDM model, their interaction with baryons are significant only in the early universe.

In this paper, we compare our theoretical predictions with Planck CMB data which is sensitive to Fourier modes, $k<0.2 \mathrm{Mpc}^{-1}$ (e.g. $[2,56]$ ). The DD model (Figure 1) also allows for significant difference in the matter power at smaller scales that cannot be probed by CMB data. The matter power at these scales can be constrained by cosmological observables such as weak lensing and the clustering of Lyman- $\alpha$ clouds (e.g. [58]) and other data such as Milky way satellite population (e.g. [54]). We hope to return to this comparison in the future.

If the dark matter particle interacts electromagnetically, it might be easier to detect it directly and it might have a bearing on the formation of stars and galaxies (see e.g. $[26,28,29,35])$. The constraints on the CCDM model from such considerations have been discussed extensively (e.g. [35]), we briefly discuss here the impact of atomic dark matter on the formation of galaxies. The observed spiral galaxies display baryonic component in a disk surrounded by a halo whose mass is dominated by dark matter. The separation of baryons from dark matter occurs because baryons can cool and therefore fall towards the center of the potential well of a virialized halo while the dark matter dominates the halo mass. The main cooling mechanisms for haloes (of primordial chemical composition) in the temperature range of $10^{4}-10^{6} \mathrm{~K}$ are line de-excitation of neutral hydrogen, line de-excitation of singly-ionized helium, and free-free emission (see e.g. [32]). For a dark matter interacting electromagnetically with baryons, we might expect dark matter to also cool and fall to the 
center of potential wells, which would be in contradiction with the observables of galaxies. Therefore, it is important to show that the dark matter atoms, and in particular the HeD and DD atoms which could constitute all the cold dark matter, do not behave as baryons for the energy scales and densities of interest in a galaxy $\left(T \simeq 10^{5} \mathrm{~K}\right.$ and $\left.n_{b} \simeq 0.01 \mathrm{~cm}^{-3}\right)$. First, these atoms cannot cool owing to any of the mechanisms discussed above because their energy scales (binding energy and atomic levels) are too large to be affected by collisions with baryons which could cause line de-excitation or ionization. Second, the time scale of collisions of $\mathrm{HeD}$ or $\mathrm{DD}$ atoms with baryons is generally larger than the age of the universe (Eqs. (2.12) and (2.15)). This implies that $\mathrm{HeD}$ and $\mathrm{DD}$ atoms behave like a non-interacting dark matter particle for typical energy scales and densities expected in a galaxy. It is possible that these particles could interact with baryons and photons in denser parts of the galaxies ${ }^{10}$ or where the energy scales are larger (e.g. supernova remnants).

The aim of this paper is to investigate the cosmological implications of a dark matter particle with electromagnetic interactions. It is possible the parameter space we studied could be further constrained from other astrophysical and experimental constraints. We leave such an investigation to a future work.

\section{Acknowledgments}

Our results are based on observations obtained with Planck (http://www.esa.int/Planck), an ESA science mission with instruments and contributions directly funded by ESA Member States, NASA, and Canada.

\footnotetext{
${ }^{10}$ The additional interaction might help in resolving the core-cusp and "too big to fail" issues as one possible way to address these discrepancies is to invoke dark matter self-interaction, e.g. see [36, 64] and references therein.
} 


\section{References}

[1] M. Ackermann et al. Measurement of separate cosmic-ray electron and positron spectra with the Fermi Large Area Telescope. Phys.Rev.Lett., 108:011103, 2012.

[2] P. A. R. Ade et al. Planck 2015 results. XIII. Cosmological parameters. Astron. Astrophys., 594:A13, 2016.

[3] P.A.R. Ade et al. Planck 2013 results. XVI. Cosmological parameters. Astron.Astrophys., 2014.

[4] O. Adriani et al. PAMELA results on the cosmic-ray antiproton flux from $60 \mathrm{MeV}$ to $180 \mathrm{GeV}$ in kinetic energy. Phys.Rev.Lett., 105:121101, 2010.

[5] M. Aguilar et al. Cosmic-ray positron fraction measurement from 1 to 30-GeV with AMS-01. Phys.Lett., B646:145-154, 2007.

[6] Z. Ahmed et al. Results from a Low-Energy Analysis of the CDMS II Germanium Data. Phys.Rev.Lett., 106:131302, 2011.

[7] D. S. Akerib, S. Alsum, H. M. Araújo, X. Bai, A. J. Bailey, J. Balajthy, P. Beltrame, E. P. Bernard, A. Bernstein, T. P. Biesiadzinski, E. M. Boulton, P. Brás, D. Byram, S. B. Cahn, M. C. Carmona-Benitez, C. Chan, A. A. Chiller, C. Chiller, A. Currie, J. E. Cutter, T. J. R. Davison, A. Dobi, J. E. Y. Dobson, E. Druszkiewicz, B. N. Edwards, C. H. Faham, S. R. Fallon, S. Fiorucci, R. J. Gaitskell, V. M. Gehman, C. Ghag, M. G. D. Gilchriese, C. R. Hall, M. Hanhardt, S. J. Haselschwardt, S. A. Hertel, D. P. Hogan, M. Horn, D. Q. Huang, C. M. Ignarra, R. G. Jacobsen, W. Ji, K. Kamdin, K. Kazkaz, D. Khaitan, R. Knoche, N. A. Larsen, C. Lee, B. G. Lenardo, K. T. Lesko, A. Lindote, M. I. Lopes, A. Manalaysay, R. L. Mannino, M. F. Marzioni, D. N. McKinsey, D.-M. Mei, J. Mock, M. Moongweluwan, J. A. Morad, A. S. J. Murphy, C. Nehrkorn, H. N. Nelson, F. Neves, K. O’Sullivan, K. C. Oliver-Mallory, K. J. Palladino, E. K. Pease, L. Reichhart, C. Rhyne, S. Shaw, T. A. Shutt, C. Silva, M. Solmaz, V. N. Solovov, P. Sorensen, S. Stephenson, T. J. Sumner, M. Szydagis, D. J. Taylor, W. C. Taylor, B. P. Tennyson, P. A. Terman, D. R. Tiedt, W. H. To, M. Tripathi, L. Tvrznikova, S. Uvarov, V. Velan, J. R. Verbus, R. C. Webb, J. T. White, T. J. Whitis, M. S. Witherell, F. L. H. Wolfs, J. Xu, K. Yazdani, S. K. Young, C. Zhang, and LUX Collaboration. Limits on Spin-Dependent WIMP-Nucleon Cross Section Obtained from the Complete LUX Exposure. Physical Review Letters, 118(25):251302, June 2017.

[8] G. Angloher, M. Bauer, I. Bavykina, A. Bento, C. Bucci, et al. Results from $730 \mathrm{~kg}$ days of the CRESST-II Dark Matter Search. Eur.Phys.J., C72:1971, 2012.

[9] E. Aprile, J. Aalbers, F. Agostini, M. Alfonsi, L. Althueser, F. D. Amaro, M. Anthony, F. Arneodo, L. Baudis, B. Bauermeister, M. L. Benabderrahmane, T. Berger, P. A. Breur, A. Brown, A. Brown, E. Brown, S. Bruenner, G. Bruno, R. Budnik, C. Capelli, J. M. R. Cardoso, D. Cichon, D. Coderre, A. P. Colijn, J. Conrad, J. P. Cussonneau, M. P. Decowski, P. de Perio, P. di Gangi, A. di Giovanni, S. Diglio, A. Elykov, G. Eurin, J. Fei, A. D. Ferella,

A. Fieguth, W. Fulgione, A. Gallo Rosso, M. Galloway, F. Gao, M. Garbini, C. Geis, L. Grandi, Z. Greene, H. Qiu, C. Hasterok, E. Hogenbirk, J. Howlett, R. Itay, F. Joerg, B. Kaminsky, S. Kazama, A. Kish, G. Koltman, H. Landsman, R. F. Lang, L. Levinson, Q. Lin, S. Lindemann, M. Lindner, F. Lombardi, J. A. M. Lopes, J. Mahlstedt, A. Manfredini, T. Marrodán Undagoitia, J. Masbou, D. Masson, M. Messina, K. Micheneau, K. Miller, A. Molinario, K. Morâ, M. Murra, J. Naganoma, K. Ni, U. Oberlack, B. Pelssers, F. Piastra, J. Pienaar, V. Pizzella, G. Plante, R. Podviianiuk, N. Priel, D. Ramírez García, L. Rauch, S. Reichard, C. Reuter, B. Riedel, A. Rizzo, A. Rocchetti, N. Rupp, J. M. F. Dos Santos, G. Sartorelli, M. Scheibelhut, S. Schindler, J. Schreiner, D. Schulte, M. Schumann, L. Scotto Lavina, M. Selvi, P. Shagin, E. Shockley, M. Silva, H. Simgen, D. Thers, F. Toschi, G. Trinchero, C. Tunnell, N. Upole, M. Vargas, O. Wack, H. Wang, Z. Wang, Y. Wei, C. Weinheimer, C. Wittweg, J. Wulf, J. Ye, Y. Zhang, T. Zhu, and Xenon Collaboration. Dark 
Matter Search Results from a One Ton-Year Exposure of XENON1T. Physical Review Letters, 121(11):111302, September 2018.

[10] E. Aprile et al. First Dark Matter Results from the XENON100 Experiment. Phys.Rev.Lett., 105:131302, 2010.

[11] R. Barkana. Possible interaction between baryons and dark-matter particles revealed by the first stars. Nature, 555:71-74, March 2018.

[12] Matthias Bartelmann and Peter Schneider. Weak gravitational lensing. Phys.Rept., 340:291-472, 2001.

[13] S.W. Barwick et al. Measurements of the cosmic ray positron fraction from 1-GeV to $50-\mathrm{GeV}$. Astrophys.J., 482:L191-L194, 1997.

[14] K.G. Begeman, A.H. Broeils, and R.H. Sanders. Extended rotation curves of spiral galaxies: Dark haloes and modified dynamics. Mon.Not.Roy.Astron.Soc., 249:523, 1991.

[15] H. A. Bethe and E. E. Salpeter. Quantum Mechanics of One-and Two-Electron Atoms. 1957.

[16] Florian Beutler et al. The clustering of galaxies in the completed SDSS-III Baryon Oscillation Spectroscopic Survey: Baryon Acoustic Oscillations in Fourier-space. Mon. Not. Roy. Astron. Soc., 2016.

[17] Diego Blas, Julien Lesgourgues, and Thomas Tram. The Cosmic Linear Anisotropy Solving System (CLASS) II: Approximation schemes. JCAP, 1107:034, 2011.

[18] K. K. Boddy, M. Kaplinghat, A. Kwa, and A. H. G. Peter. Atomic dark matter with hyperfine interactions. In American Institute of Physics Conference Series, volume 1900 of American Institute of Physics Conference Series, page 040002, November 2017.

[19] J. D. Bowman, A. E. E. Rogers, R. A. Monsalve, T. J. Mozdzen, and N. Mahesh. An absorption profile centred at 78 megahertz in the sky-averaged spectrum. Nature, 555:67-70, March 2018.

[20] Michael Boylan-Kolchin, James S. Bullock, and Manoj Kaplinghat. Too big to fail? The puzzling darkness of massive Milky Way subhaloes. Mon.Not.Roy.Astron.Soc., 415:L40, 2011.

[21] T. Brinckmann, J. Lesgourgues, B. Audren, K. Benabed, and S. Prunet. MontePython 3: Parameter inference code for cosmology. Astrophysics Source Code Library, May 2018.

[22] L. Chuzhoy and E. W. Kolb. Reopening the window on charged dark matter. JCAP, 7:014, July 2009.

[23] J. M. Cline, Z. Liu, and W. Xue. Millicharged atomic dark matter. Phys. Rev. D, 85(10):101302, May 2012.

[24] Douglas Clowe, Marusa Bradac, Anthony H. Gonzalez, Maxim Markevitch, Scott W. Randall, Christine Jones, and Dennis Zaritsky. A direct empirical proof of the existence of dark matter. Astrophys. J., 648:L109-L113, 2006.

[25] Nathaniel Craig and Andrey Katz. The Fraternal WIMP Miracle. JCAP, 1510(10):054, 2015.

[26] Francis-Yan Cyr-Racine and K. Sigurdson. Cosmology of atomic dark matter. Phys. Rev. D, 87(10):103515, May 2013.

[27] W. J. G. de Blok. The Core-Cusp Problem. Adv. Astron., 2010:789293, 2010.

[28] A. de Rújula, S. L. Glashow, and U. Sarid. Charged dark matter. Nuclear Physics B, 333:173-194, March 1990.

[29] S. Dimopoulos, D. Eichler, R. Esmailzadeh, and G. D. Starkman. Getting a charge out of dark matter. Phys. Rev. D, 41:2388-2397, April 1990.

[30] S. Dodelson. Modern cosmology. Academic Press, 2003. 
[31] A.D. Dolgov and S.H. Hansen. Massive sterile neutrinos as warm dark matter. Astropart.Phys., 16:339-344, 2002.

[32] B. T. Draine. Physics of the Interstellar and Intergalactic Medium. Princeton University Press, 2011.

[33] S. L. Dubovsky, D. S. Gorbunov, and G. I. Rubtsov. Narrowing the window for millicharged particles by CMB anisotropy. JETP Lett., 79:1-5, 2004. [Pisma Zh. Eksp. Teor. Fiz.79,3(2004)].

[34] Cora Dvorkin, Kfir Blum, and Marc Kamionkowski. Constraining Dark Matter-Baryon Scattering with Linear Cosmology. Phys. Rev., D89(2):023519, 2014.

[35] A. Fialkov, R. Barkana, and A. Cohen. Constraining Baryon-Dark-Matter Scattering with the Cosmic Dawn 21-cm Signal. Physical Review Letters, 121(1):011101, July 2018.

[36] Robert Foot and Sunny Vagnozzi. Solving the small-scale structure puzzles with dissipative dark matter. Journal of Cosmology and Astroparticle Physics, 2016(07):013âĂŞ013, Jul 2016.

[37] Patrick J. Fox, Roni Harnik, Joachim Kopp, and Yuhsin Tsai. Missing Energy Signatures of Dark Matter at the LHC. Phys.Rev., D85:056011, 2012.

[38] Shea Garrison-Kimmel, Michael Boylan-Kolchin, James S. Bullock, and Evan N. Kirby. Too Big to Fail in the Local Group. 2014.

[39] Jessica Goodman, Masahiro Ibe, Arvind Rajaraman, William Shepherd, Tim M.P. Tait, et al. Constraints on Light Majorana dark Matter from Colliders. Phys.Lett., B695:185-188, 2011.

[40] G. Hinshaw et al. Nine-Year Wilkinson Microwave Anisotropy Probe (WMAP) Observations: Cosmological Parameter Results. Astrophys.J.Suppl., 208:19, 2013.

[41] L. Hui, J. P. Ostriker, S. Tremaine, and E. Witten. Ultralight scalars as cosmological dark matter. Phys. Rev. D, 95(4):043541, February 2017.

[42] Y. I. Izotov, T. X. Thuan, and N. G. Guseva. A new determination of the primordial He abundance using the He I $\lambda 10830 \AA$ emission line: cosmological implications. Mon. Not. Roy. Astron. Soc., 445:778-793, November 2014.

[43] Ayuki Kamada, Kazunori Kohri, Tomo Takahashi, and Naoki Yoshida. Effects of electrically charged dark matter on cosmic microwave background anisotropies. Physical Review D, 95(2), Jan 2017.

[44] M. Y. Khlopov. Dark atoms of dark matter from new stable quarks and leptons. In J. Kouneiher, C. Barbachoux, T. Masson, and D. Vey, editors, American Institute of Physics Conference Series, volume 1446 of American Institute of Physics Conference Series, pages 99-110, June 2012.

[45] Anatoly A. Klypin, Andrey V. Kravtsov, Octavio Valenzuela, and Francisco Prada. Where are the missing Galactic satellites? Astrophys.J., 522:82-92, 1999.

[46] K. Kohri and T. Takahashi. Cosmology with long-lived charged massive particles. Physics Letters B, 682:337-341, January 2010.

[47] Ely D. Kovetz, Vivian Poulin, Vera Gluscevic, Kimberly K. Boddy, Rennan Barkana, and Marc Kamionkowski. Tighter limits on dark matter explanations of the anomalous EDGES $21 \mathrm{~cm}$ signal. Phys. Rev., D98(10):103529, 2018.

[48] L. D. Landau and E. M. Lifshitz. Quantum mechanics. Course of theoretical physics, Oxford: Pergamon Press, 1965, 1965.

[49] L. D. Landau and E. M. Lifshitz. Mechanics. 1969.

[50] C.-P. Ma and E. Bertschinger. Cosmological Perturbation Theory in the Synchronous and Conformal Newtonian Gauges. Astrophys. J., 455:7, December 1995. 
[51] B. Moore, S. Ghigna, F. Governato, G. Lake, Thomas R. Quinn, et al. Dark matter substructure within galactic halos. Astrophys.J., 524:L19-L22, 1999.

[52] V. Mukhanov. "CMB-Slow" or How to Determine Cosmological Parameters by Hand? International Journal of Theoretical Physics, 43:623-668, March 2004.

[53] V. Mukhanov. Physical Foundations of Cosmology. November 2005.

[54] Ethan O. Nadler, Vera Gluscevic, Kimberly K. Boddy, and Risa H. Wechsler. Constraints on dark matter microphysics from the milky way satellite population. The Astrophysical Journal, 878(2):L32, Jun 2019.

[55] Julio F. Navarro, Carlos S. Frenk, and Simon D. M. White. The Structure of cold dark matter halos. Astrophys. J., 462:563-575, 1996.

[56] Planck Collaboration, N. Aghanim, Y. Akrami, M. Ashdown, J. Aumont, C. Baccigalupi, M. Ballardini, A. J. Banday, R. B. Barreiro, N. Bartolo, S. Basak, R. Battye, K. Benabed, J.-P. Bernard, M. Bersanelli, P. Bielewicz, J. J. Bock, J. R. Bond, J. Borrill, F. R. Bouchet, F. Boulanger, M. Bucher, C. Burigana, R. C. Butler, E. Calabrese, J.-F. Cardoso, J. Carron, A. Challinor, H. C. Chiang, J. Chluba, L. P. L. Colombo, C. Combet, D. Contreras, B. P. Crill, F. Cuttaia, P. de Bernardis, G. de Zotti, J. Delabrouille, J.-M. Delouis, E. Di Valentino, J. M. Diego, O. Doré, M. Douspis, A. Ducout, X. Dupac, S. Dusini, G. Efstathiou, F. Elsner, T. A. Enßlin, H. K. Eriksen, Y. Fantaye, M. Farhang, J. Fergusson, R. Fernandez-Cobos, F. Finelli, F. Forastieri, M. Frailis, E. Franceschi, A. Frolov, S. Galeotta, S. Galli, K. Ganga, R. T. Génova-Santos, M. Gerbino, T. Ghosh, J. González-Nuevo, K. M. Górski, S. Gratton, A. Gruppuso, J. E. Gudmundsson, J. Hamann, W. Handley, D. Herranz, E. Hivon, Z. Huang, A. H. Jaffe, W. C. Jones, A. Karakci, E. Keihänen, R. Keskitalo, K. Kiiveri, J. Kim, T. S. Kisner, L. Knox, N. Krachmalnicoff, M. Kunz, H. Kurki-Suonio, G. Lagache, J.-M. Lamarre, A. Lasenby, M. Lattanzi, C. R. Lawrence, M. Le Jeune, P. Lemos, J. Lesgourgues, F. Levrier, A. Lewis, M. Liguori, P. B. Lilje, M. Lilley, V. Lindholm, M. López-Caniego, P. M. Lubin, Y.-Z. Ma, J. F. Macías-Pérez, G. Maggio, D. Maino, N. Mandolesi, A. Mangilli,

A. Marcos-Caballero, M. Maris, P. G. Martin, M. Martinelli, E. Martínez-González, S. Matarrese, N. Mauri, J. D. McEwen, P. R. Meinhold, A. Melchiorri, A. Mennella, M. Migliaccio, M. Millea, S. Mitra, M.-A. Miville-Deschênes, D. Molinari, L. Montier, G. Morgante, A. Moss, P. Natoli, H. U. Nørgaard-Nielsen, L. Pagano, D. Paoletti, B. Partridge, G. Patanchon, H. V. Peiris, F. Perrotta, V. Pettorino, F. Piacentini, L. Polastri, G. Polenta, J.-L. Puget, J. P. Rachen, M. Reinecke, M. Remazeilles, A. Renzi, G. Rocha, C. Rosset, G. Roudier, J. A. Rubiño-Martín, B. Ruiz-Granados, L. Salvati, M. Sandri, M. Savelainen, D. Scott, E. P. S. Shellard, C. Sirignano, G. Sirri, L. D. Spencer, R. Sunyaev, A.-S. Suur-Uski, J. A. Tauber, D. Tavagnacco, M. Tenti, L. Toffolatti, M. Tomasi, T. Trombetti, L. Valenziano, J. Valiviita, B. Van Tent, L. Vibert, P. Vielva, F. Villa, N. Vittorio, B. D. Wandelt, I. K. Wehus, M. White, S. D. M. White, A. Zacchei, and A. Zonca. Planck 2018 results. VI. Cosmological parameters. ArXiv e-prints, July 2018.

[57] G.B. Rybicki and A.P. Lightman. Radiative processes in astrophysics. New York, Wiley-Interscience, 1979. 393 p, 1979.

[58] Abir Sarkar, Subinoy Das, and Shiv K. Sethi. How Late can the Dark Matter form in our universe? JCAP, 1503(03):004, 2015.

[59] Abir Sarkar, Rajesh Mondal, Subinoy Das, Shiv.K. Sethi, Somnath Bharadwaj, and David J. E. Marsh. The effects of the small-scale DM power on the cosmological neutral hydrogen (HI) distribution at high redshifts. JCAP, 1604(04):012, 2016.

[60] Jonathan L. Sievers et al. The Atacama Cosmology Telescope: Cosmological parameters from three seasons of data. JCAP, 1310:060, 2013.

[61] Kris Sigurdson and Marc Kamionkowski. Charged - particle decay and suppression of small scale power. Phys. Rev. Lett., 92:171302, 2004. 
[62] Max Tegmark et al. Cosmological parameters from SDSS and WMAP. Phys.Rev., D69:103501, 2004.

[63] Max Tegmark et al. Cosmological Constraints from the SDSS Luminous Red Galaxies. Phys.Rev., D74:123507, 2006.

[64] S. Tulin and H.-B. Yu. Dark matter self-interactions and small scale structure. Physics Reports, 730:1-57, February 2018.

[65] Matteo Viel, Julien Lesgourgues, Martin G. Haehnelt, Sabino Matarrese, and Antonio Riotto. Constraining warm dark matter candidates including sterile neutrinos and light gravitinos with WMAP and the Lyman-alpha forest. Phys.Rev., D71:063534, 2005.

[66] Matthew G. Walker and Jorge Penarrubia. A Method for Measuring (Slopes of) the Mass Profiles of Dwarf Spheroidal Galaxies. Astrophys. J., 742:20, 2011.

[67] Weishuang Linda Xu, Cora Dvorkin, and Andrew Chael. Probing sub-GeV Dark Matter-Baryon Scattering with Cosmological Observables. Phys. Rev., D97(10):103530, 2018. 